\title{
Article \\ Genomic Diversity of Bacteriophages Infecting the Genus Acinetobacter
}

\author{
Hugo Oliveira ${ }^{1} \mathbb{D}$, Rita Domingues ${ }^{1}$, Benjamin Evans ${ }^{2} \mathbb{D}$, J. Mark Sutton ${ }^{3} \mathbb{D}$, Evelien M. Adriaenssens ${ }^{4} \mathbb{D}$ \\ and Dann Turner $5, *$ (iD
}

Citation: Oliveira, H.; Domingues, R.; Evans, B.; Sutton, J.M.; Adriaenssens, E.M.; Turner, D. Genomic Diversity of Bacteriophages Infecting the Genus Acinetobacter. Viruses 2022, 14, 181. https://doi.org/10.3390/v14020181 Academic Editor: Mikael Skurnik Received: 14 December 2021 Accepted: 10 January 2022 Published: 19 January 2022

Publisher's Note: MDPI stays neutral with regard to jurisdictional claims in published maps and institutional affiliations.

Copyright: (C) 2022 by the authors. Licensee MDPI, Basel, Switzerland. This article is an open access article distributed under the terms and conditions of the Creative Commons Attribution (CC BY) license (https:// creativecommons.org/licenses/by/ $4.0 /)$.
1 Centre of Biological Engineering, University of Minho, Campus de Gualtar Braga, 4710-057 Braga, Portugal; hugooliveira@deb.uminho.pt (H.O.); ritadomingues1898@gmail.com (R.D.)

2 Norwich Medical School, University of East Anglia, Norwich NR4 7TJ, UK; benjamin.evans@uea.ac.uk

3 United Kingdom Health Security Agency, Research and Evaluation, Porton Down, Salisbury SP4 OJG, UK; mark.sutton@phe.gov.uk

4 Quadram Institute Bioscience, Norwich Research Park, Norwich NR4 7UQ, UK; evelien.adriaenssens@quadram.ac.uk

5 Department of Applied Sciences, Faculty of Health and Applied Sciences, University of the West of England, Bristol BS16 1QY, UK

* Correspondence: dann2.turner@uwe.ac.uk

\begin{abstract}
The number of sequenced Acinetobacter phage genomes in the International Nucleotide Sequence Database Collaboration has increased significantly in recent years, from 37 in 2017 to a total of 139 as of January 2021 with genome sizes ranging from 31 to $378 \mathrm{~kb}$. Here, we explored the genetic diversity of the Acinetobacter phages using comparative genomics approaches that included assessment of nucleotide similarity, shared gene content, single gene phylogeny, and the networkbased classification tool vConTACT2. Phages infecting Acinetobacter sp. are genetically diverse and can be grouped into 8 clusters (subfamilies) and 46 sub-clusters (genera), of which 8 represent genomic singletons (additional genera). We propose the creation of five new subfamilies and suggest a reorganisation of the genus Obolenskvirus. These results provide an updated view of the viruses infecting Acinetobacter species, providing insights into their diversity.
\end{abstract}

Keywords: Acinetobacter; bacteriophages; comparative genomics; phage therapy; bioinformatics

\section{Introduction}

Advances in sequencing technologies and bioinformatics tools have enabled in-depth exploration and revealed the vastness of bacteriophage (phage) biodiversity. The diversity of phages is exemplified by the number of novel genes, little nucleotide sequence similarities between many sequenced phages, and, increasingly, by the sheer number of distinct viral sequences being uncovered through metaviromics of various environmental niches [1].

Comparative genomics has emerged as a fundamental tool for the investigation of phage genomes, allowing the identification of related phage genomes and providing an opportunity to identify and evaluate relationships both within and between clades [2]. Genome-based metrics are now used by the Bacterial Viruses Subcommittee of the International Committee on the Taxonomy of Viruses (ICTV) for the delineation and assignment of formal taxonomic ranks [3]. Comparisons at the nucleotide sequence and whole proteome levels have shown that, for lytic phages at least, evolution of these viruses appears to be predominantly vertical and that some clades of phages represent ancient lineages (e.g., the N4-like phages of the family Schitoviridae), given that closely related phages have been isolated from disparate geographic locations, environments, and times.

Acinetobacter baumannii is an opportunistic pathogen which is associated with a range of nosocomial infections. Due to an ability to persist for long periods in the environment and to develop resistance to multiple antibiotic classes, including broad spectrum beta-lactams such as third generation cephalosporins and carbapenems, fluoroquinolones, 
aminoglycosides, tigecycline, and colistin, extensively drug-resistant and even pan-drug resistant strains are emerging in clinical settings worldwide [4,5]. Infections caused by $A$. baumannii are more common in intensive care units and surgical wards, manifesting as ventilator-associated pneumonia or bacteraemia but also as soft-tissue injury and urinary tract and gastrointestinal infections [6,7].

Members of the genus Acinetobacter are defined phenotypically as Gram negative, catalase positive, oxidase negative, non-fermenting coccobacilli. There are currently 69 species recognised within the genus that are validly published and possess a correct name (https://lpsn.dsmz.de/genus/Acinetobacter, accessed on 21 June 2021). A. baumannii is part of the Acinetobacter calcoaceticus-baumannii (Acb) complex, which includes species with similar phenotypic and genotypic properties. The Acb complex is comprised of five pathogenic species: A. baumannii, Acinetobacter pittii, Acinetobacter nosocomialis, Acinetobacter seifertii, and Acinetobacter dijkshoorniae, as well as the non-pathogenic species Acinetobacter calcoaceticus [8].

Although strains of A. baumannii show limited diversity among core genes, the genome is both plastic and dynamic, which is shown by significant variance in accessory gene content. A significant factor in the accessory genome variance is the acquisition of a wide variety of mobile genetic elements as well as hotspots of recombination, which has contributed to the evolution of extensive and pan-drug resistant phenotypes [9]. Notably, comparative analysis of isolates from international clonal lineage I has shown hotspots of recombination associated with capsular polysaccharide, lipooligosaccharide, and outer membrane proteins-surface structures which are commonly targeted by phages for adsorption $[10,11]$.

The rapid accumulation of resistance genes has severely depleted the number of antibiotics available as effective treatment options for infections caused by the Acb complex. Increasingly, resistance to carbapenems and polymixins threatens to further compound this problem [12]. Collectively, this has resulted in the designation of A. baumannii as a critical target for the development of new therapeutic options by the World Health Organisation (WHO) (https://www.who.int/medicines/publications/WHO-PPL-Short_ Summary_25Feb-ET_NM_WHO.pdf, accessed on 21 June 2021). With the lack of new antibiotics in development, the use of phages (phage therapy) or phage-derived proteins as a therapeutic option, often applied as a combinatorial therapy with existing classes of antibiotics, is gaining increased attention. As importantly, the investigation and analysis of phage genomes and gene products can lead to the development of novel molecular tools to study this important pathogen.

In 2017, there were 37 Acinetobacter phage genomes available in public databases. Based on the number of shared proteins, nucleotide identity, and genomic architecture, the Acinetobacter viral population was comprised of six discrete clusters and two singletons [13]. Notably, half of the protein-coding genes had no assigned functions, illustrating the vast amount of the so-called "genetic dark matter" in these viral genomes. Since then, the number of available Acinetobacter phage genomes has increased substantially, necessitating a re-evaluation of these data. Here, we present an updated analysis of all sequenced Acinetobacter phages held in the International Nucleotide Sequence Database Collaboration (INSDC) as of the 21st of January 2021. The Acinetobacter phage genomes were re-annotated and examined using a comparative bioinformatics approach based upon whole genome alignments, protein clustering, and phylogenetic analysis. We highlight the taxonomic population structure of these phages and identify core, accessory, and unique proteincoding genes present within, and shared between, clades.

\section{Materials and Methods}

\subsection{Genome Annotation}

Acinetobacter phage genomes in the FASTA file format were downloaded from GenBank (21 January 2021). Genome sequences were compared to each other using BLASTN and edited so that they were colinear by taking the reverse complement and/or reopening the 
sequence. To ensure consistency in gene calls for downstream annotation and analyses, all genomes were re-annotated using Prokka [14] using a custom 'Caudovirales' database (http:/ / millardlab.org/bioinformatics/lab_server/phage-genome-annotation/, accessed on 21 January 2021). For each annotated genome, translated ORF sequences were searched against hidden Markov models (HMM) profiles downloaded from the VOG database (release number vog204) [15] using hmmscan with an e-value cut-off of $1 \times 10^{-5}$. Matches to $p$ VOG profiles were considered significant at an e-value of $\leq 1 \times 10^{-5}$ with $\geq 35 \%$ coverage of the profile HMM.

\subsection{Global Profiling of Acinetobacter Phage Genomes}

To provide a snapshot of the relationship between the Acinetobacter phages and the wider phage population, a gene-sharing network of all phage genomes held in GenBank (24 January 2021) was constructed using the INPHARED pipeline [16] and vConTACT2 0.9.19, using DIAMOND v2.0.7.145 was applied for protein clustering and ClusterOne v1.0 for protein and viral cluster analyses. The resulting network was visualised using Cytoscape (version 3.8.2) [17] and annotated with Adobe Illustrator (Adobe Inc., San Jose, CA, USA).

\subsection{Genome Clustering}

The diversity of Acinetobacter phage genomes was assessed using DNA sequence comparisons, protein clustering, and single-gene phylogenies. At the DNA level, VIRIDIC was used for an all-versus-all nucleotide sequence comparison of the genomes with the following parameters: species threshold 95, genus threshold 65, -word_size 7, -reward 2, -penalty -3, -gapopen 5, and -gapextend 2 [18].

For the identification of orthologous groups of proteins, two approaches were used. First, translated ORF sequences were clustered into groups using PIRATE [19] by using parameters of $30-40 \%$ minimum sequence identity and $\geq 50 \%$ coverage of high-scoring pairs. Amino acid sequences for each group of proteins were extracted and further functional inferences obtained using InterProScan version 5.48-83.0 [20]. Sequences comprising of protein groups were first aligned using Clustal Omega version 1.2.4 [21] and then used to search the UniRef30_2020_06 database using HHblits with a single iteration and an e-value threshold of $1 \times 10^{-5}$. Alignments produced by HHblits were converted to hmm profiles using the tool hhmake and then queried against the pdb70_from_the mmcif_210127 database using HHsearch. Remote homologies identified using HHsearch were used to further refine the phage genome annotations where the results were considered significant if the probability was greater than $75 \%$ and the e-value was $<1 \times 10^{-3}$.

As a secondary measure, Acinetobacter phage genomes were also analysed with Phamerator to sort genes into phamilies of related sequences (phams if shared by at least two proteins; orphams for unique proteins) using kclust alignments [22]. A structured query language (SQL) database containing the phage genome GenBank files was created and imported to Phamerator using a Virtual Machine (VM Virtual Box, Oracle Corporation, Austin, TX, USA). Next, SplitsTree (version 5_2_28-beta) [23] was used to plot the genome diversity based on their shared protein content, which was obtained by Phamerator.

The maximum likelihood phylogenetic trees were reconstructed using IQ-Tree version 1.6.12 [24] from alignments generated using Clustal Omega, and implemented the fast method of the substitution model selection with ModelFinder, SH-aLRT test and ultrafast bootstrap approximation (UFBoot) with 1000 replicates [25-27]. The phylogenetic trees were visualised using ITOL [28]. The comparative genome diagrams were prepared using Clinker [29], using a BLASTp identity threshold of 30\% and edited in Adobe Illustrator (Adobe Inc., San Jose, CA, USA).

\subsection{Cluster Assignment}

Here, we employ the terms cluster, to represent the subfamily-level relationships, and sub-cluster, to denote genera using the guidelines of the Bacterial Viruses Subcommittee of the International Committee on the Taxonomy of Viruses (ICTV). Phages were grouped 
into clusters (subfamilies) based on a minimum of $30 \%$ shared proteins identified using PIRATE and assigned a letter to represent each individual cluster. These clusters were further divided into sub-clusters (genera) using a threshold of $\geq 65 \%$ nucleotide sequence similarity and assigned a number-for example, cluster A, sub-cluster 1 (A1). Below these thresholds, genomes identified as being unrelated to other Acinetobacter phages or to the wider population of sequenced phages were assigned as genomic singletons (unique genera).

\subsection{Transmission Electron Microscopy}

Phages RPH5R, RPH2R, TRS2, and TRS1 were isolated from wastewater treatment plants. Virions were pelleted from PEG-precipitated or $\mathrm{CsCl}$ purified samples by centrifugation at $25,000 \times g$ for $65 \mathrm{~min}$. Pellets were resuspended in $100 \mu \mathrm{L}$ of $0.1 \mathrm{M}$ ammonium acetate (pH7.2). Specimens for transmission electron microscopy were prepared by the addition of $5 \mu \mathrm{L}$ to 400 mesh carbon-coated formvar grids and stained by the addition of an equal volume of $2 \% w / v$ uranyl acetate. Grids were examined using a Phillips CM10 equipped with a Gatan Orius SC1000 CCD camera (Gatan, Pleasanton, CA, USA).

\section{Results}

\subsection{Taxonomy of the Acinetobacter Phages}

A dataset of 134 Acinetobacter phage genomes was prepared (Table S1) after the exclusion of four phages, PBAB08 [MG366114], PBAB25 [MG366115], $\varphi$ FG02 [MT648818], and $\varphi \mathrm{C} 001$ [MT648819], due to the presence of multiple frameshift assembly errors. The single representative of the class Leviviricetes, species Appevirus quebecense, AP205 was also excluded.

To assess the relationships among the Acinetobacter phages at the nucleotide sequence level, intergenomic similarities were calculated reciprocally between pairs of viral genomes using VIRIDIC [18]. Using the ICTV recommended criteria for the demarcation of genera [30], we identified a total of 46 sub-clusters based on a threshold of a $\geq 65 \%$ nucleotide sequence similarity (Figure S1). The Acinetobacter phages can therefore be classified into 46 genera, of which 14 correspond to the ratified ICTV taxa: Zedzedvirus (A1), Lazarusvirus (A2), Hadassahvirus (A3), Lasallevirus (A4), Acajnonavirus (A5), Obolenskvirus (B4), Saclayvirus (C3), Friunavirus (E2), Pettyvirus (E3), Daemvirus (E4), Vieuvirus (F3), Lokivirus (G1), Presleyvirus (H1), Exceevirus (H2), and Metrivirus (singleton ME3).

Several of the individual sub-clusters exhibit a nucleotide sequence similarity of $>30 \%$, but below the recommended genus threshold of $65-70 \%$, suggesting that subfamily level relationships may exist between them. While nucleotide sequence similarity provides a robust measure for closely related phages, the examination of shared protein content provides a measure of the intra-cluster variability, identification of signature genes, and reveals more distant relationships at the subfamily level or higher. To assess these relationships, a pan-genome analysis of the 15,734 proteins encoded by the Acinetobacter phages was performed using PIRATE and Phamerator. Using PIRATE, proteins were clustered using the criteria of a $>30 \%$ sequence identity and a $>50 \%$ query coverage. A total of 1492 groups, consisting of two or more proteins and 2444 unique proteins, were identified using this approach (File S1). Phamerator identified 1650 phams of two or more proteins and 2825 orphams (64\%) composed of unique sequences. We define (i) core proteins as those that are present in all members of a cluster or sub-cluster; (ii) accessory proteins as those present in two or more, but not all of the members; and (iii) unique proteins as those found only in a single phage genome.

Hierarchical clustering of the shared protein content obtained from the Phamerator and PIRATE pan-genome analyses demonstrated that 33 of the sub-clusters could be grouped to form a total of 8 clusters (A, B, C, E, F, H, L, and M) representing subfamily level relationships that exhibited, at minimum, 11 conserved core genes (Figure 1 and Table 1). At present, only two of these clusters are recognised as formal ICTV taxa, the subfamilies Twarogvirinae (Cluster A) and Beijerinckvirinae (Cluster E). While no wider relationships 
within the Acinetobacter phages were identified for five of the sub-clusters (D1, G1, I1, J1, and K1), it is possible that they may be assigned subfamily status with the isolation of related phages in the future. The remaining eight phages represented single species that exhibited no significant similarity in nucleotide or shared protein content and are assigned as genomic singletons.

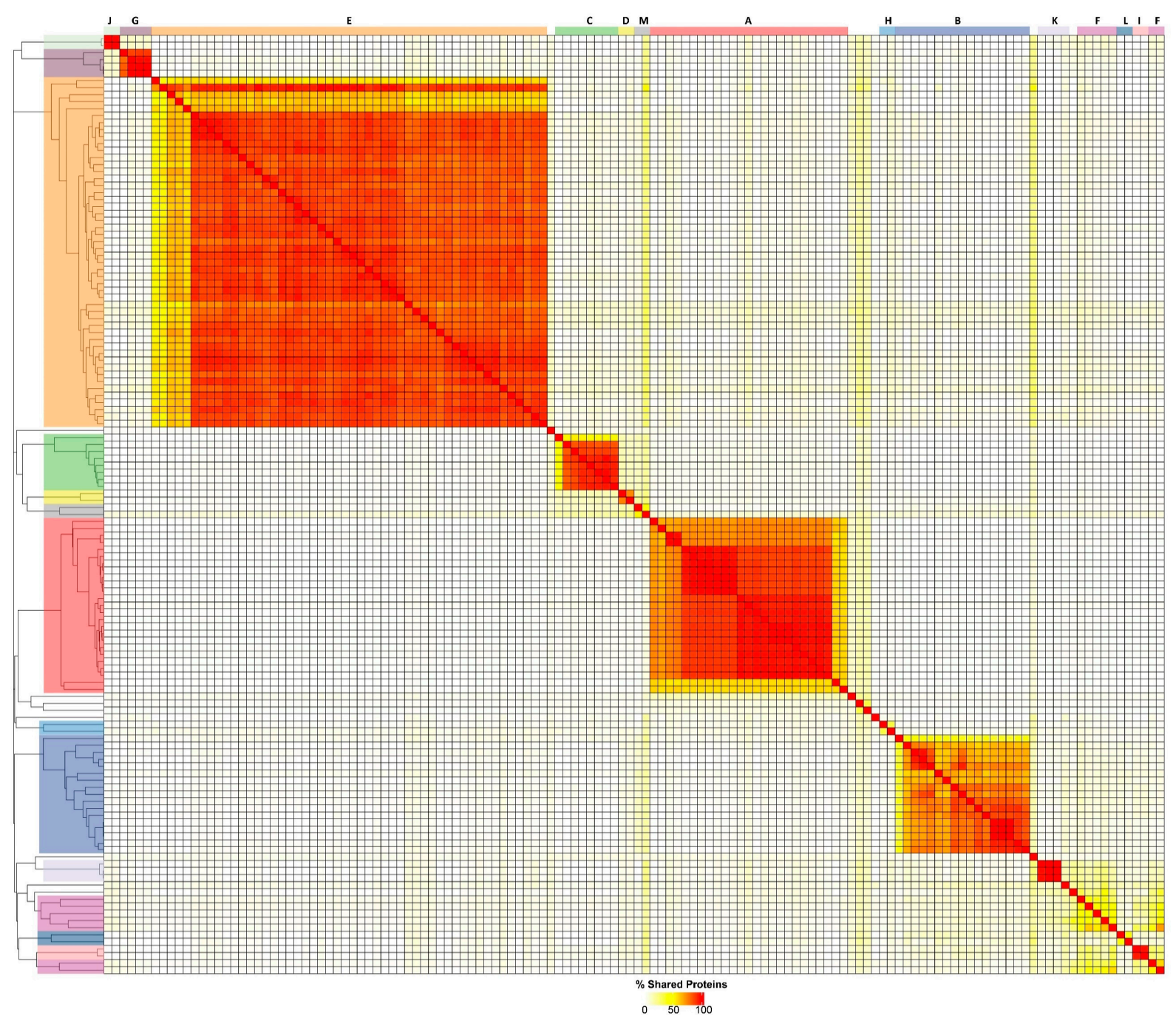

Figure 1. Heatmap of the percentage of proteins shared between pairs of Acinetobacter phage genomes. The heatmap was produced from the output of the PIRATE pan-genome analysis and hierarchically clustered using the complete linkage method in R. Clusters are indicated by coloured blocks at the top of the heatmap and within the dendrogram according to the key: $\odot \mathrm{A} ; \bigcirc \mathrm{B} ; \odot \mathrm{C} ; \odot \mathrm{D} ; \odot \mathrm{E} ; \odot \mathrm{F}$; $\mathrm{G} ; \bigcirc \mathrm{H} ; \bigcirc \mathrm{I}$ J; $\bigcirc \mathrm{K} \odot \mathrm{L}$; $\mathrm{M}$. 
Table 1. Diversity of Acinetobacter phage genomes, composed of 8 clusters, 38 sub-clusters, and 8 singletons. Core, accessory, and unique protein groups identified within each cluster and subcluster are presented. Clusters corresponding to subfamilies are presented in bold type. Numbers in parentheses refer to protein groups found exclusively within the designated cluster. Ratified ICTV taxa are presented in italics, while proposed subfamily and genus names are presented as roman text within quotations.

\begin{tabular}{|c|c|c|c|c|c|c|}
\hline Cluster & Taxonomy & $\begin{array}{c}\text { Representative } \\
\text { Phage }\end{array}$ & No. of Genomes & $\begin{array}{c}\text { No. of Core } \\
\text { Protein Groups }\end{array}$ & $\begin{array}{l}\text { No. of Accessory } \\
\text { Protein Groups }\end{array}$ & $\begin{array}{l}\text { No. of Unique } \\
\text { Proteins }\end{array}$ \\
\hline A & Twarogvirinae & - & 25 & $107(81)$ & $255(225)$ & 252 \\
\hline A1 & Zedzedvirus & ZZ1 & 2 & $241(52)$ & $\mathrm{N} / \mathrm{A}$ & 4 \\
\hline A2 & Lasarusvirus & Lazarus & 12 & $218(11)$ & $32(22)$ & 2 \\
\hline $\mathrm{A} 3$ & Hassadvirus & PhT2 & 7 & $227(13)$ & $26(17)$ & $\overline{3}$ \\
\hline A4 & Lasallevirus & Acj61 & 1 & $\mathrm{~N} / \mathrm{A}$ & $\mathrm{N} / \mathrm{A}$ & 46 \\
\hline A5 & Acajnonavirus & Acj9 & 1 & $\mathrm{~N} / \mathrm{A}$ & $\mathrm{N} / \mathrm{A}$ & 46 \\
\hline A6 & "Freretvirus" & Ac42 & 1 & $\mathrm{~N} / \mathrm{A}$ & $\mathrm{N} / \mathrm{A}$ & 74 \\
\hline A7 & "Audubonvirus" & 133 & 1 & $\mathrm{~N} / \mathrm{A}$ & $\mathrm{N} / \mathrm{A}$ & 77 \\
\hline B & "Zhukovskyvirinae" & - & 17 & $23(22)$ & $117(100)$ & 63 \\
\hline B1 & "Solivirus" & phiAC-1 & 1 & $\mathrm{~N} / \mathrm{A}$ & $\mathrm{N} / \mathrm{A}$ & 33 \\
\hline $\mathrm{B} 2$ & "Fengtaivirus" & IME-AB2 & 8 & $56(4)$ & $36(4)$ & 6 \\
\hline B3 & "Shapingbavirus" & $\mathrm{AbP} 2$ & 5 & $58(1)$ & $42(33)$ & 5 \\
\hline $\mathrm{B} 4$ & Obolenskvirus & AP22 & 1 & N/A & $\mathrm{N} / \mathrm{A}$ & 3 \\
\hline B5 & "Wenzhouvirus" & AB1 & 1 & $\mathrm{~N} / \mathrm{A}$ & $\mathrm{N} / \mathrm{A}$ & 8 \\
\hline B6 & "Dongdavirus" & IME284 & 1 & $\mathrm{~N} / \mathrm{A}$ & $\mathrm{N} / \mathrm{A}$ & 9 \\
\hline $\mathrm{C}$ & "Astridvirinae" & - & 8 & $69(42)$ & $117(106)$ & 88 \\
\hline $\mathrm{C} 1$ & "Acibelquatrovirus" & Acibel004 & 1 & $\mathrm{~N} / \mathrm{A}$ & $\mathrm{N} / \mathrm{A}$ & 58 \\
\hline $\mathrm{C} 2$ & "Powislevirus" & phiAbaA1 & 1 & $\mathrm{~N} / \mathrm{A}$ & $\mathrm{N} / \mathrm{A}$ & 15 \\
\hline $\mathrm{C} 3$ & Saclayvirus & TAC1 & 6 & $129(97)$ & $32(28)$ & 15 \\
\hline D1 & "Caradocvirus" & AM24 & 2 & $115(87)$ & $\mathrm{N} / \mathrm{A}$ & 45 \\
\hline E* & Beijernickvirinae & - & 49 & $21(20)$ & $57(45)$ & 69 \\
\hline E1 & "Aristophanvirus" & Aristophanes & 1 & $\mathrm{~N} / \mathrm{A}$ & $\mathrm{N} / \mathrm{A}$ & 15 \\
\hline $\mathrm{E} 2$ * & Friunavirus & Fri1 & 45 & $27(0)$ & $70(61)$ & 15 \\
\hline E3 & Pettyvirus & Petty & 1 & $\mathrm{~N} / \mathrm{A}$ & $\mathrm{N} / \mathrm{A}$ & 12 \\
\hline E4 & Daemvirus & Acibel007 & 1 & $\mathrm{~N} / \mathrm{A}$ & $\mathrm{N} / \mathrm{A}$ & 13 \\
\hline E5 & "Lisbonvirus" & F1245-05 & 1 & $\mathrm{~N} / \mathrm{A}$ & $\mathrm{N} / \mathrm{A}$ & 14 \\
\hline $\mathbf{F}$ & "Junivirinae" & - & 7 & $11(2)$ & 87 & 96 \\
\hline $\mathrm{F} 1$ & "Shemyakinvirus" & AM106 & 1 & $\mathrm{~N} / \mathrm{A}$ & $\mathrm{N} / \mathrm{A}$ & 16 \\
\hline F2 & "Bogotavirus" & Ab11510-phi & 1 & $\mathrm{~N} / \mathrm{A}$ & $\mathrm{N} / \mathrm{A}$ & 8 \\
\hline F3 & Vieuvirus & Bphi-B1251 & 2 & $44(12)$ & $\mathrm{N} / \mathrm{A}$ & 17 \\
\hline $\mathrm{F} 4$ & "Breacavirus" & RPH5R & 1 & $\mathrm{~N} / \mathrm{A}$ & $\mathrm{N} / \mathrm{A}$ & 19 \\
\hline F5 & "Geihvirus" & Ab105-2phi & 1 & $\mathrm{~N} / \mathrm{A}$ & $\mathrm{N} / \mathrm{A}$ & 18 \\
\hline F6 & "Reipivirus" & Ab105-3phi & 1 & $\mathrm{~N} / \mathrm{A}$ & $\mathrm{N} / \mathrm{A}$ & 18 \\
\hline G1 & Lokivirus & Loki & 4 & $43(38)$ & $10(9)$ & 5 \\
\hline $\mathbf{H}$ & Schitoviridae & - & 2 & $28(28)$ & N/A & 104 \\
\hline H1 & Presleyvirus & Presley & 1 & $\mathrm{~N} / \mathrm{A}$ & $\mathrm{N} / \mathrm{A}$ & 54 \\
\hline $\mathrm{H} 2$ & Xceevirus & XC38 & 1 & $\mathrm{~N} / \mathrm{A}$ & $\mathrm{N} / \mathrm{A}$ & 50 \\
\hline I1 & "Xubiasvirus" & Ab105-1phi & 2 & $58(33)$ & $\mathrm{N} / \mathrm{A}$ & 4 \\
\hline $\mathrm{J} 1$ & "Stillvirus" & DMU1 & 2 & $53(41)$ & $\mathrm{N} / \mathrm{A}$ & 2 \\
\hline K1 & "Haartmanvirus" & fEg-Aba01 & 3 & $47(32)$ & $2(1)$ & 0 \\
\hline $\mathbf{L}$ & "Grainevirinae" & - & 2 & $21(18)$ & N/A & 26 \\
\hline L1 & "Corvusvirus" & RPH2R & 1 & $\mathrm{~N} / \mathrm{A}$ & $\mathrm{N} / \mathrm{A}$ & 14 \\
\hline L2 & "Boudicavirus" & TRS2 & 1 & $\mathrm{~N} / \mathrm{A}$ & $\mathrm{N} / \mathrm{A}$ & 14 \\
\hline $\mathbf{M}$ & "Soothillvirinae" & - & 2 & $79(53)$ & N/A & 129 \\
\hline M1 & "Bragavirus" & B9 & 1 & $\mathrm{~N} / \mathrm{A}$ & $\mathrm{N} / \mathrm{A}$ & 56 \\
\hline M2 & "Bathrowvirus" & BS46 & 1 & $\mathrm{~N} / \mathrm{A}$ & $\mathrm{N} / \mathrm{A}$ & 73 \\
\hline Singleton & Metrivirus & ME3 & 1 & $\mathrm{~N} / \mathrm{A}$ & $\mathrm{N} / \mathrm{A}$ & 261 \\
\hline Singleton & "Gogmagogvirus" & TRS5 & 1 & $\mathrm{~N} / \mathrm{A}$ & $\mathrm{N} / \mathrm{A}$ & 405 \\
\hline Singleton & "Comoranvirus" & $\mathrm{BFG}$ & 1 & $\mathrm{~N} / \mathrm{A}$ & $\mathrm{N} / \mathrm{A}$ & 422 \\
\hline Singleton & "Lianhecunvirus" & $5 \mathrm{~W}$ & 1 & $\mathrm{~N} / \mathrm{A}$ & $\mathrm{N} / \mathrm{A}$ & 22 \\
\hline Singleton & "Fengtaivirus" & ABPH49 & 1 & $\mathrm{~N} / \mathrm{A}$ & $\mathrm{N} / \mathrm{A}$ & 246 \\
\hline Singleton & "Camillusvirus" & TRS1 & 1 & $\mathrm{~N} / \mathrm{A}$ & $\mathrm{N} / \mathrm{A}$ & 23 \\
\hline Singleton & "Jiatongvirus" & SH-Ab 15599 & 1 & $\mathrm{~N} / \mathrm{A}$ & $\mathrm{N} / \mathrm{A}$ & 127 \\
\hline Singleton & "Lucanusvirus" & Arae & 1 & $\mathrm{~N} / \mathrm{A}$ & $\mathrm{N} / \mathrm{A}$ & 55 \\
\hline
\end{tabular}

* Number of coding sequences calculated after the exclusion of the partial genome of phage AB3.

The relationships between the Acinetobacter phages were illustrated by a network phylogeny created using the entire complement of proteins, which was converted to a binary matrix to denote the presence or absence of each protein within each individual phage genome. The network reflected the assignment of sub-clusters from the nucleotide sequence comparisons and revealed the more distant subfamily level relationships between some clusters, which are represented by a deeper branching structure (Figure 2). 
(A)

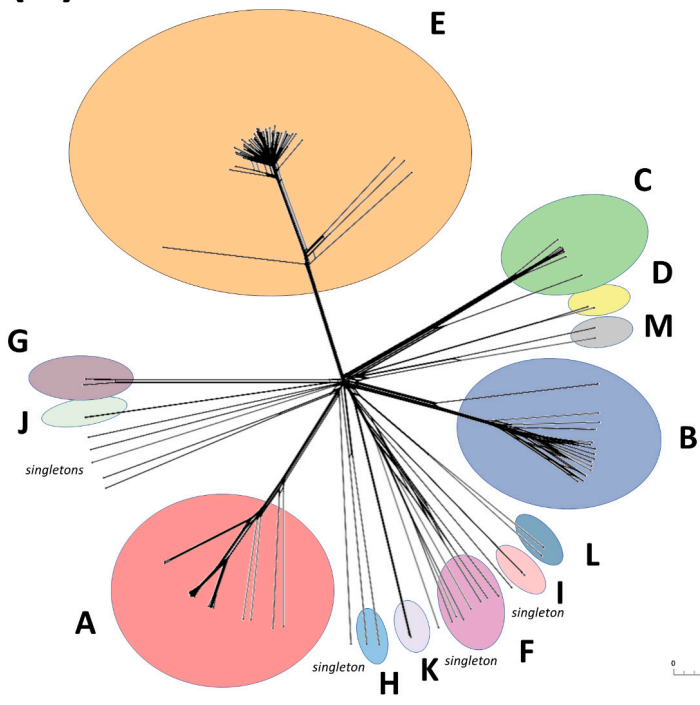

(B)

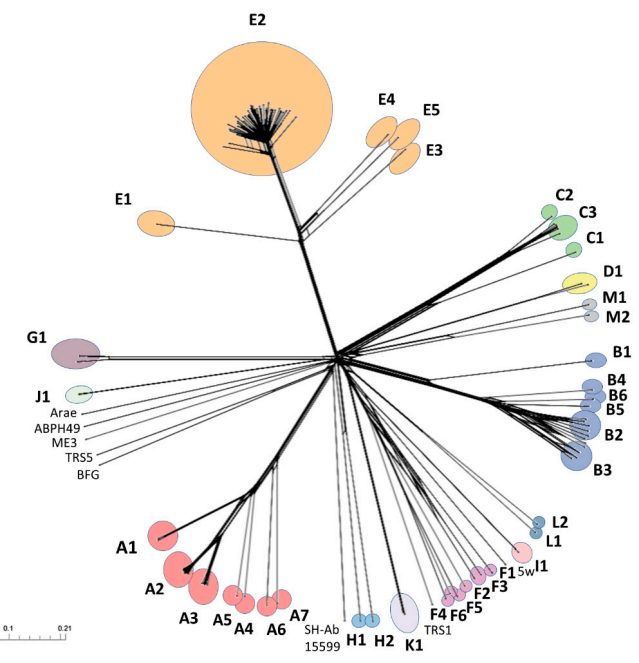

Figure 2. Diversity of Acinetobacter phage genomes. From a total of 15,734 predicted genes of the 134 Acinetobacter phage genomes, it generated 1650 phams of two or more proteins and 2825 orphams $(64 \%)$ composed of unique sequences were identified in Phamerator. The resulting network was to visualize the genomics diversity with SplitsTree using "genome content distance" mode. Phages were assigned to (A) clusters and (B) subclusters if sharing > 35 genes or lower, respectively. The scale bar indicates 0.01 substitution per site (number of gene differences, presence or absence, per gene site). Clusters and subclusters are marked by ellipses and bold letters according to the key: $\mathrm{A} ; \bigcirc \mathrm{B} ; \bigcirc \mathrm{C}$;

$\mathrm{D} ; \bigcirc \mathrm{E} ; \bigcirc \mathrm{F} ; \bigcirc \mathrm{G} ; \bigcirc \mathrm{H} ; \odot \mathrm{I} ; \bigcirc \mathrm{J} ; \bigcirc \mathrm{K} \odot \mathrm{L} ; \bigcirc \mathrm{M}$. Singleton phages Arae, ABP49, ME3, TRS5, BFG and $\mathrm{SH}-\mathrm{Ab} 15599$ are named in (B).

With the exception of cluster F, which is discussed below, the assignment of subfamily level clusters was also supported by a low standard deviation of the average genome size, $\% \mathrm{G}+\mathrm{C}$ content, and the number of protein coding genes, as well as by phylogenetic analysis of the major capsid and portal vertex proteins (Figure 3).

vContact2 was used to assess the relationship of the Acinetobacter phages with the wider phage population (Figure 4). In the resulting network, the Acinetobacter phages fell into groups that reflect those defined by their nucleotide similarity and shared protein content, but more distant relationships were also revealed. Members of the genus Lokivirus (sub-cluster G1) formed part of a larger clade of siphoviruses that include members of the genera Septimatrevirus, Kilunavirus, and Titanvirus. Singleton phages SH-Ab 15599 and ABPH49 clustered with members of the Ackermannviridae and Vequintavirinae, respectively. The sub-clusters representing the temperate Acinetobacter phages fell within a much larger grouping that mainly consisted of phages capable of lysogeny.

The number of sequenced Acinetobacter phages has increased substantially since the last comparative analysis of phages infecting this bacterial genus, revealing a diverse and heterogeneous population [13]. While existing phage genera have expanded to include new members, many distinct new species representatives have been isolated. In addition to the ICTV-ratified subfamilies Twarogvirinae and Beijerinckvirinae, we propose the creation of five new subfamilies, and suggest a reorganisation of the genus Obolenskvirus by splitting this genus and creating a new subfamily to encompass the new genera. Herein, short descriptions of each of the major clusters (subfamilies) and their constituent sub-clusters (genera) are provided. We also focus on three themes: host adsorption, nucleotide modifications, and the lysis systems of these diverse groups of phages. 
(A)

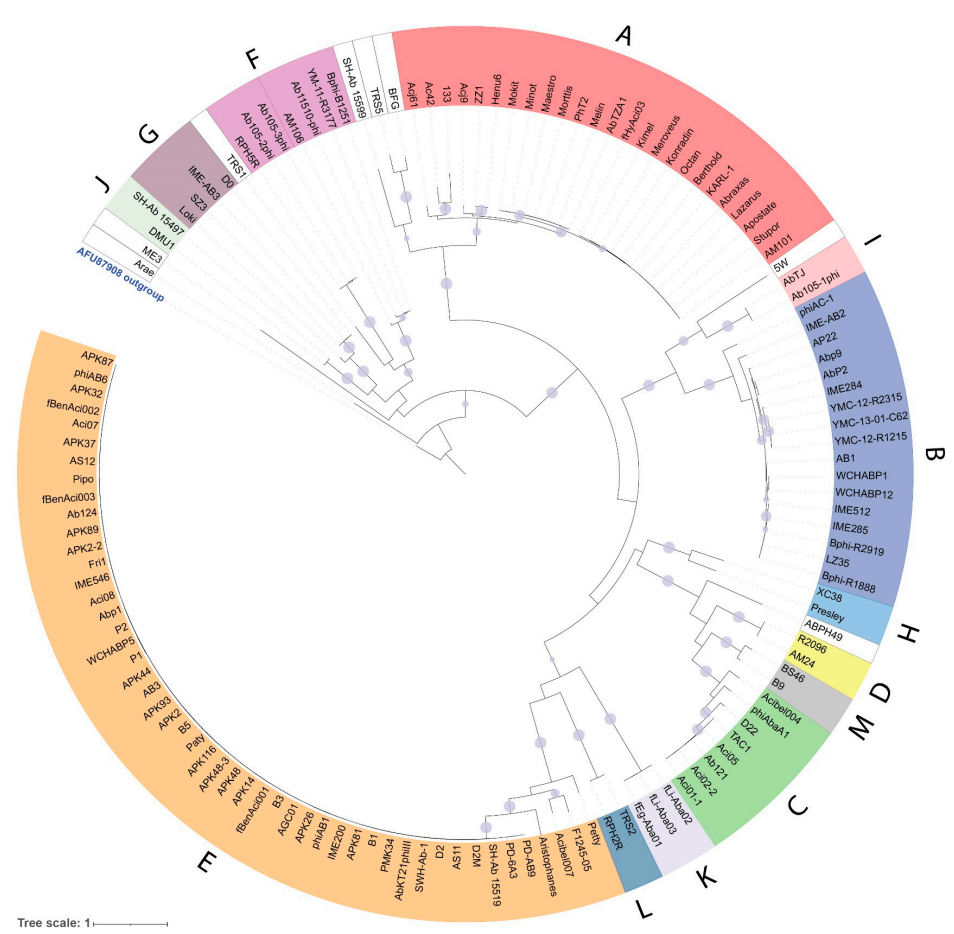

(B)

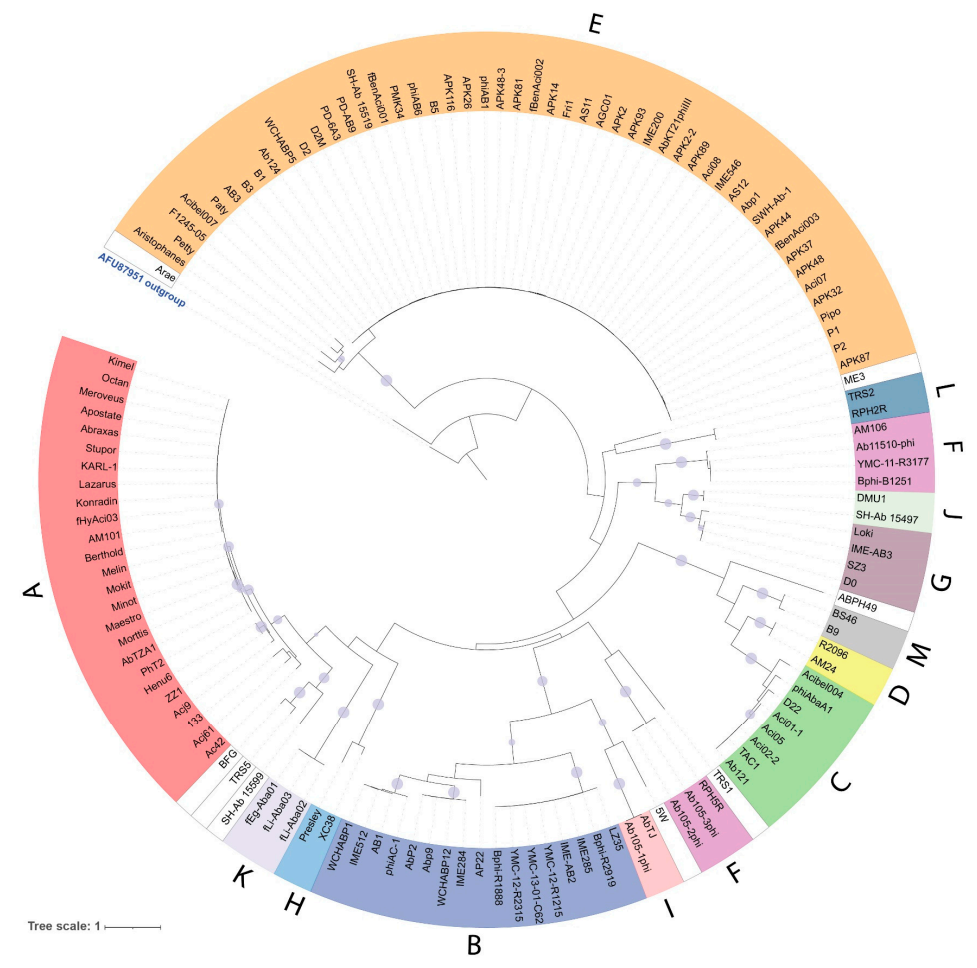

Figure 3. Phylogenetic analysis of (A) the portal vertex and (B) major capsid proteins of the Acinetobacter phages. The maximum likelihood phylogenetic trees were inferred from alignments generated by Clustal Omega with VT + F + R3 (portal) and Q.pfam + F + G4 (capsid) models of evolution and 1000 bootstrap replicates and SH-Alrt test using IQ-TREE and visualised using ITOL. The trees were rooted using the portal or major capsid protein from Caulobacter phage CcrColossus, indicated by bold blue type. Ultrafast bootstrap values ( $\geq 95 \%)$ are marked with filled circles, with the size proportional to the bootstrap value. Clusters are denoted by coloured arcs and labels on the outer edge of the tree $(\odot \mathrm{A} ; \bigcirc \mathrm{B} ; \odot \mathrm{C} ; \bigcirc \mathrm{D} ; \odot \mathrm{E} ; \odot \mathrm{F} ; \bigcirc \mathrm{G} ; \bigcirc \mathrm{H} ; \odot \mathrm{I} ; \bigcirc \mathrm{J} ; \bigcirc \mathrm{K} ; \bigcirc \mathrm{L} ; \bigcirc \mathrm{M})$. The scale bar represents substitutions per site. 


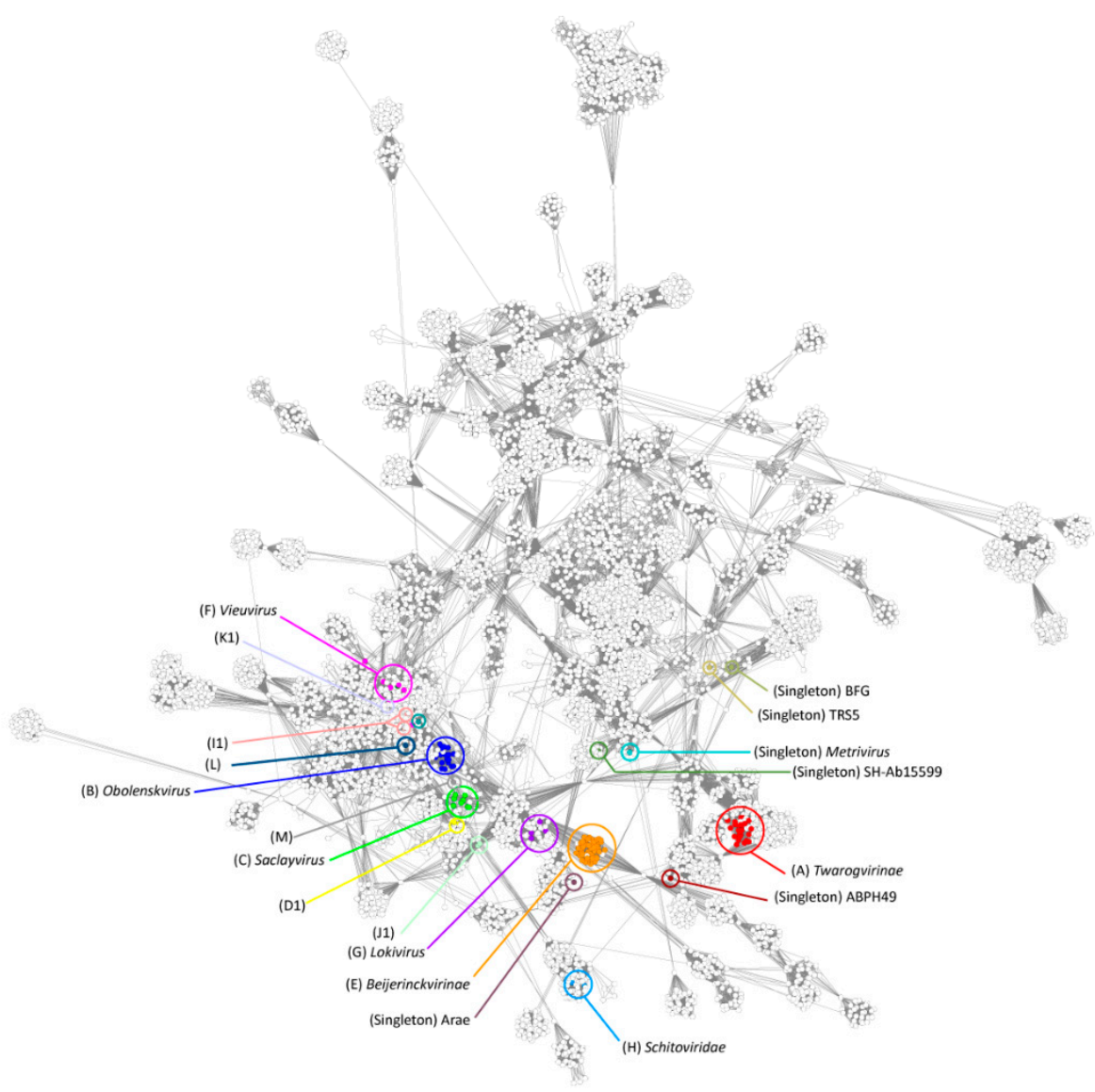

Figure 4. vConTACT2 network analysis. The network represents a subset of dsDNA prokaryotic virus genomes. Each circle (node) represents a genome and connecting lines (edges) represent the similarity between genomes based on shared clusters of proteins. Acinetobacter phage genomes are highlighted as filled coloured nodes with cluster designations provided in parentheses, and ICTV-ratified genus and subfamily names are presented in italics. Clusters are coloured according to the key: A; $\bigcirc \mathrm{B} ; \bigcirc \mathrm{C} ; \bigcirc \mathrm{D} 1 ; \bigcirc \mathrm{E} ; \bigcirc \mathrm{F} ; \bigcirc \mathrm{G} ; \bigcirc \mathrm{H} ; \bigcirc \mathrm{I} ; \bigcirc \mathrm{J1} ; \bigcirc \mathrm{K} ; \bigcirc \mathrm{L} ; \bigcirc \mathrm{M} ;$ Singleton phages: BFG; TRS5; 5W; ABPH49; TRS1; SH-Ab 15599; Arae; ME3.

\subsection{T4-like Phages Infecting Acinetobacter sp.}

Cluster $\mathrm{A}$ is comprised of 25 T4-like phages that that can be grouped into seven sub-clusters (A1-A7) that infect the host species A. baumannii, A. johnsonnnii, and A. pittii. The taxonomy of the T4-like phages has recently undergone a significant re-evaluation, resulting in the creation of two families, Straboviridae and Kyanoviridae (ICTV taxonomic proposal 2021.082B.A.v1). All of the T4-like Acinetobacter phages are classified within the subfamily Twarogvirinae of the Straboviridae. These phages exhibit a typical T4-like virion morphology, which comprises of a prolate head and a contractile tail that terminates in a baseplate with long and short tail fibers. At minimum, these phages exhibit $22 \%$ nucleotide sequence similarity but share a total of 107 core and 255 accessory protein groups, of which 81 and 225, respectively, are unique to the Twarogvirinae and are not found in any other cluster of Acinetobacter phages.

\subsection{Phages of the Obolenskvirus Represent a New Subfamily of Myoviruses}

Cluster B has six sub-clusters (genera B1-B6). It contains Acinetobacter phages currently classified within the genus Obolenskvirus and a single outlier, phiAC-1, that shares an average of $38.5 \%$ of its coding sequences and $25-31 \%$ sequence similarity with members of this cluster. Here we propose that the 17 cluster B phages represent a discrete subfamily consisting of 15 phage species. The genomes exhibit a common modular and syntenic 
organization with a total of 21 core proteins present across the subfamily, predominantly representing the virion structural and assembly proteins (Figure 5).

B2

B2

B2

B2

B2

B2

B2

B2

B3

B3

B3

B3

B3

B4

B5

B6
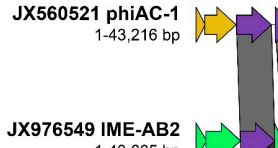

JX976549 IME-AB2 $1-43,665$ bp

KJ817802 YMC-13-01-C62 $1-44,844$ bp

KP861229 YM11/12/R2315 $1-44,846$ bp

KP861231 YM11/12/R1215 14,866 bp

KU510289 LZ35
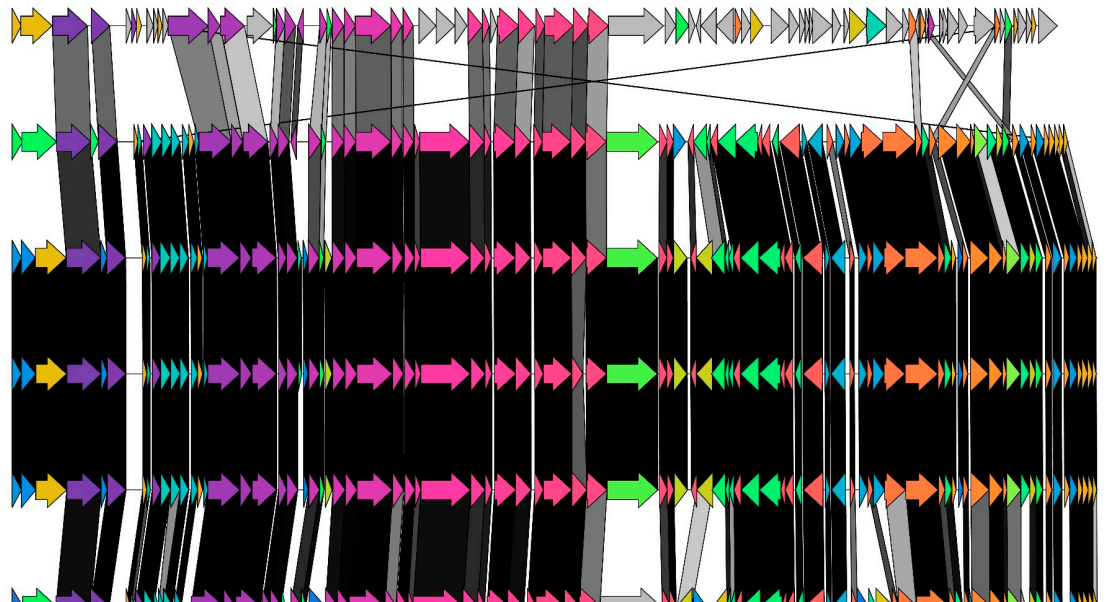

(1)

MH853786 vB_AbaM_IME285
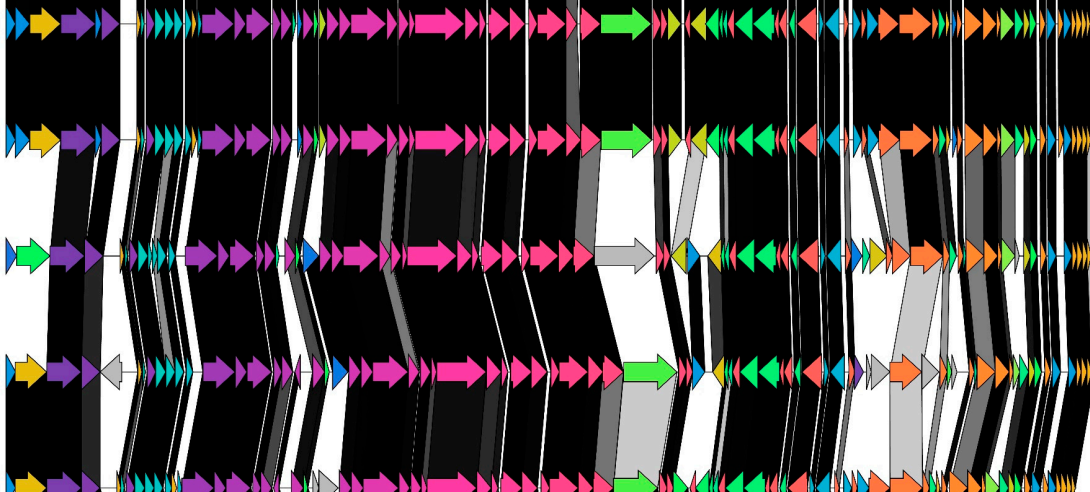

MN516421 Bphi-R2919 $1-44,227$ bp

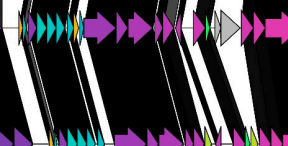
MN516422 Bphi-R1888
$1-44,590$ bp
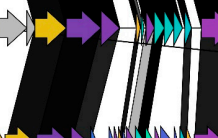
KY670595 WCHABP12
$1-45,415$ bp
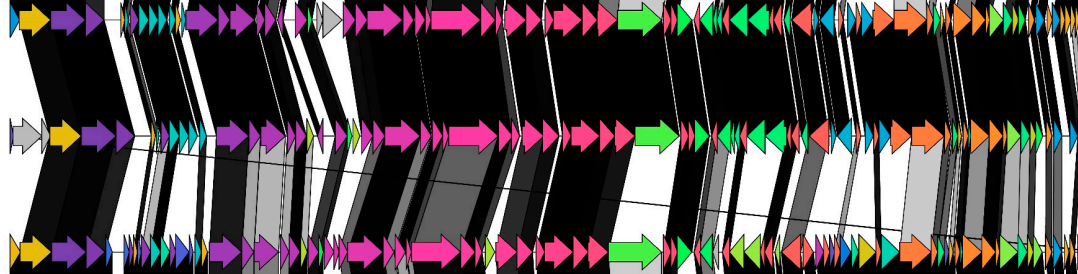

KY829116 WCHABP1

1-45,888 bp
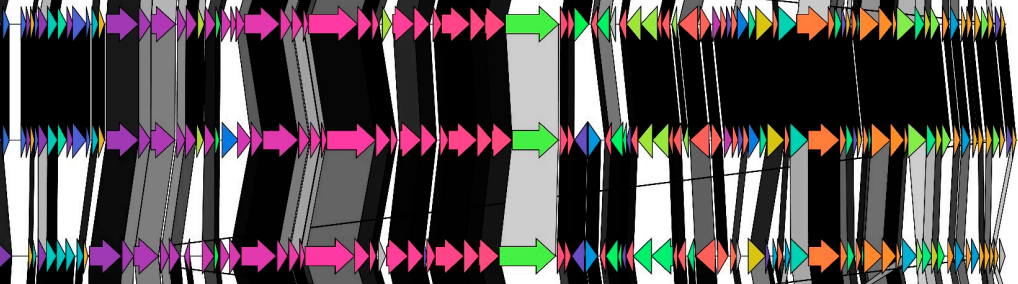

MF346584 AbP2
$1-45,373$ bp

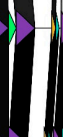

MH853788 vB_AbaM_IME512

1-46,499 bp
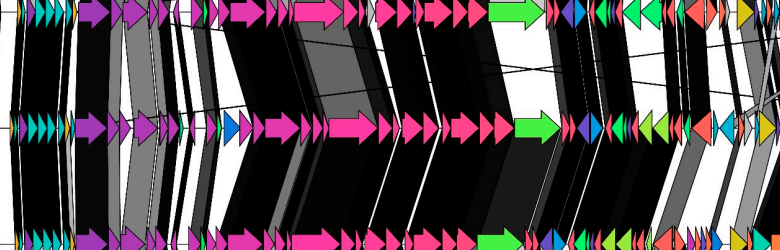

N)

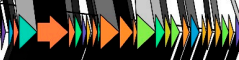

MN166083 Ab

$1-44,820$ bp
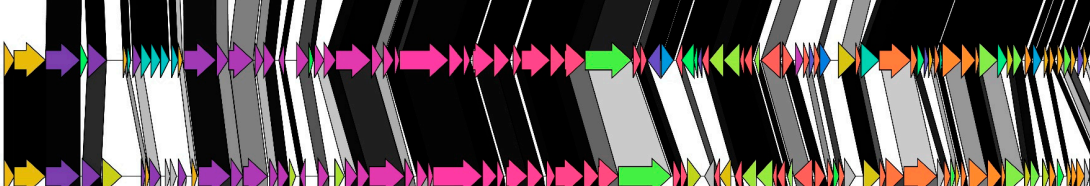

HE806280 AP22
$1-46,387$
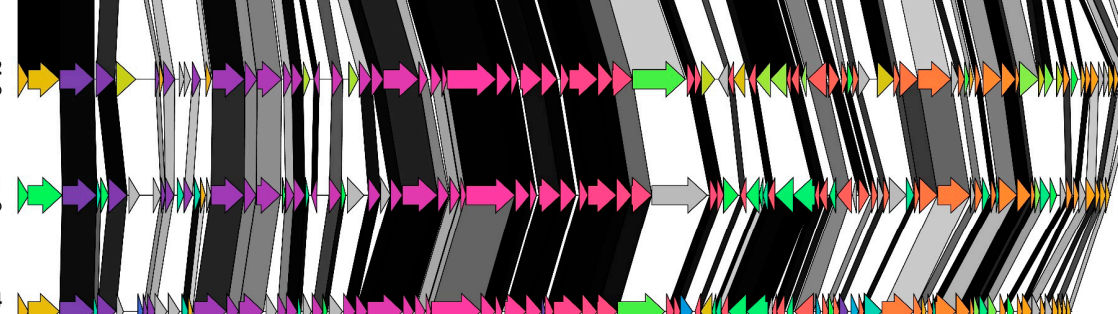

HM368260 AB1
$1-45,161$ bp

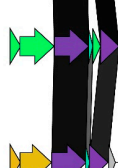

MH853787 vB_AbaM_IME284

$$
\text { 1-43,557 bp }
$$

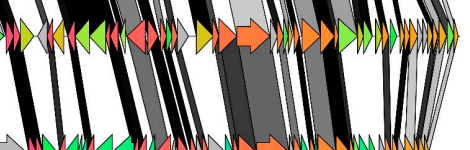

Figure 5. Comparative genome alignment of phages comprising sub-cluster B. Phage genomes are presented alongside their designated sub-cluster, accession number, name and genome length. Coding sequences are represented by arrows, coloured to reflect homologous groups identified by Clinker, and are linked by grey bars shaded to represent the percentage amino acid identity, as indicated in the legend. 
One interesting feature is that, in all members of this cluster, the virion structure and assembly module is separated between the head morphogenesis protein and prohead protease by several ORFs of unknown function that exhibit the differences in composition between the member species. While there is a high degree of shared protein content (55.8-93.6\% of ORFs) between each of the 17 phages in Cluster B, we noted that the nucleotide similarity falls below the recommended ICTV threshold of $65-70 \%$. By this criterion, the Obolenskvirus could be split into six separate genera.

\subsection{The Cluster Comprising Saclayvirus, Acibel004, and phiAbaA1 Represent a New Subfamily} of Myoviruses

Cluster $\mathrm{C}$ is comprised of eight myoviruses (Acibel004, phiAbaA1, D22, TAC1, B09_Aci011, B09_Aci02-2, B09_Aci05, and Ab121) separated into three sub-clusters (genera C1-C3). These phages possess genomes ranging from 99.7 to $104.4 \mathrm{~kb}$ with 151 to 173 protein coding genes in a syntenic and modular arrangement (Figure 6).
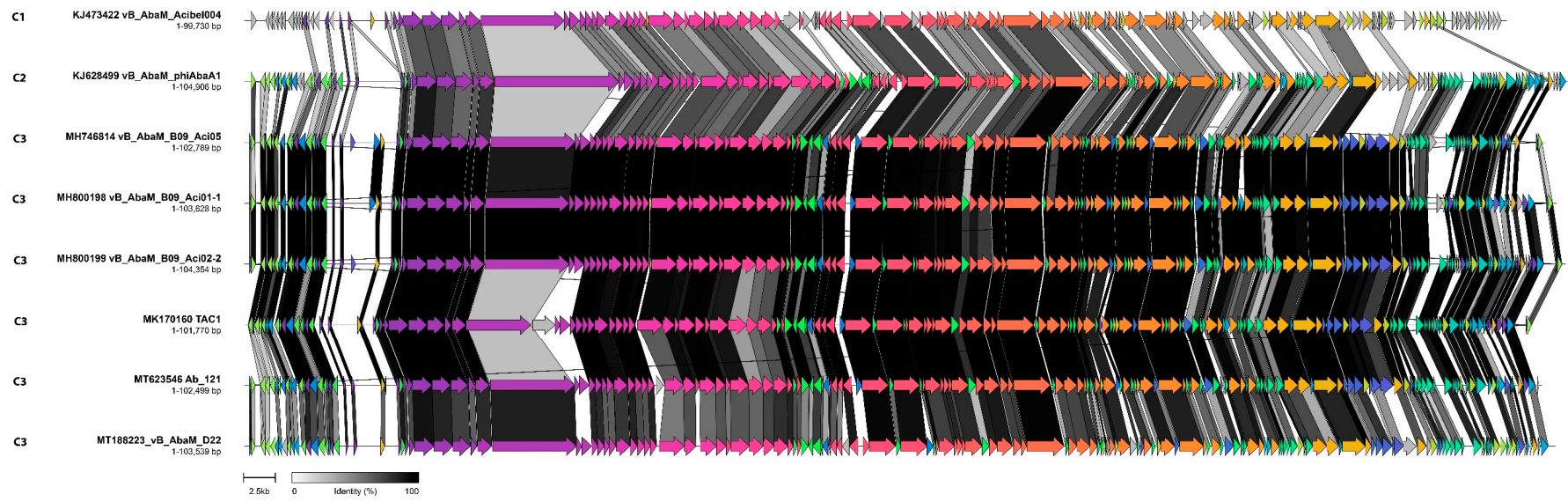

Figure 6. Comparative genome alignment of Acinetobacter phages comprising cluster C. Phage genomes are presented alongside their designated sub-cluster, accession number, name and genome length. Coding sequences are represented by arrows, coloured to reflect homologous groups identified by Clinker, and are linked by grey bars shaded to represent the percentage amino acid identity, as indicated in the legend.

Transmission electron micrographs of Acibel004 and B09_Aci05 show a common morphotype with a characteristic distal tail structure appearing to show a triangular cluster of tail fibers that converge at the apex [31,32]. At the nucleotide sequence level, Acibel004 (sub-cluster C1) and phiAbaA1 (sub-cluster C2) can be classed as outliers, exhibiting a $\sim 30 \%$ and $60 \%$ sequence similarity and an average similarity of 49.5 and $80.5 \%$ in their proteins, respectively, when compared to other members of cluster $C$. The remaining phages, TAC1, D22, B09_Aci01-1, B09_Aci02-2, B09_Aci05, and Ab121 (sub-cluster C3) formed a monophyletic clade characterised by an 83-94\% sequence similarity and 129 core proteins shared. Three of these phages, B09_Aci05, B09_Aci01-1, and B09_Aci02-2 are currently classified within the genus Saclayvirus (ICTV Taxonomic Proposal 2019.072B). Given that there are 69 core protein groups shared across these eight phages, we propose the creation of a new subfamily tentatively named as the "Astridvirinae", after Queen Astrid Military Hospital where Acibel004 was first isolated.

\subsection{AM24 and R2096 Represent a New Genus of Myoviruses}

Phages YMC13/03/R2096 (R2096) and AM24 remain the only two members of cluster D1. This grouping was supported by a $78.8 \%$ nucleotide sequence similarity and $70.2 \%$ shared proteins (Figure 7). Of the 115 core proteins, 87 were exclusive to this cluster, and these two species differ in gene content within the early and genome replication modules. Both phages have a myovirus morphology, identical genome organisation, and 
form plaques with opaque halos, which are indicative of depolymerase activity. AM24 has been shown to exhibit specificity for the K9 capsule type $[33,34]$.

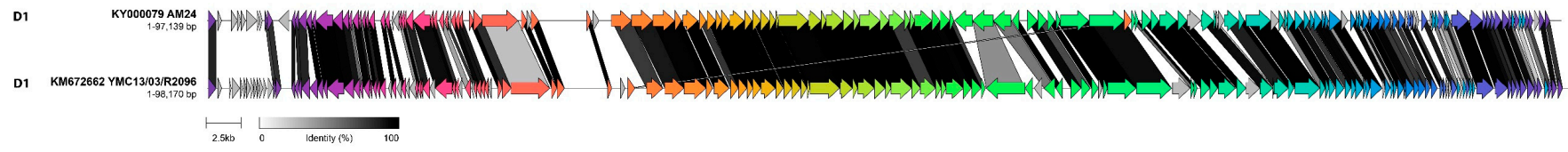

Figure 7. Comparative genome alignment of phages comprising sub-cluster D1. Phage genomes are presented alongside their designated sub-cluster, accession number, name and genome length. Coding sequences are represented by arrows, coloured to reflect homologous groups identified by Clinker, and are linked by grey bars shaded to represent the percentage amino acid identity, as indicated in the legend.

\subsection{Expansion of the Subfamily Beijerinckvirinae}

Cluster E represented the largest group of phages infecting Acinetobacter sp., comprising five sub-clusters (genera E1-E5). They are members of the Autographiviridae subfamily Beijerinckvirinae that has now expanded to include a total of 50 isolates. They are podoviruses that possess small genomes (c. $40 \mathrm{~kb}$ ) with short direct terminal repeats and encode a DNA-dependent RNA polymerase, which is characteristic of the family Autographiviridae. Sub-cluster E2, delineated as the genus Friunavirus, represents the largest and most conserved genus of Acinetobacter phages, comprising of 47 highly similar genomes sharing a $>70 \%$ sequence similarity and $>70 \%$ shared proteins. These phages differ primarily at the middle and C-terminal regions of the tailspike protein responsible for polysaccharide depolymerase activities, in the complement of some putative early genes, and the presence or absence of homing endonucleases. We noted that two phages, Pipo [MW366783] and Paty [MW366784], while described as Klebsiella phages in GenBank, are more likely to be Acinetobacter phages given the degree of nucleotide similarity and shared protein content with constituent members of the Friunavirus. A further four isolates, Acibel007 (sub-cluster E4), Aristophanes (sub-cluster E1), F1254-05 (sub-cluster E5), and Petty (subcluster E3), represent new species in individual genera within the subfamily, exhibiting a $\sim 25 \%$ sequence similarity to members of the Friunavirus and sharing 21 core proteins when the partial genome of AB3 is excluded.

\subsection{Temperate Acinetobacter Phages Encode Diverse Complements of Accessory Proteins}

The number of isolated or induced temperate Acinetobacter phages has expanded in recent years. Previously, cluster F was comprised of two phages, Bphi-B1251 and YMC11/11/R3177 (R3177), which we now define as sub-cluster F3 and represents the members of the genus Vieuvirus. Relative to Bphi-B1251, R3177 exhibits a $62.4 \%$ sequence similarity and has $56.4 \%$ shared proteins, where the difference is accounted for by the localised differences in the gene content, which suggests these two related phages might undertake different lifestyles [13]. A further five siphoviruses, AM106, Ab11510-phi RPH5R, Ab105-2phi, and Ab105-3phi (sub-clusters F1, F2, F4, F5 and F6, respectively), exhibit a degree of sequence similarity and shared protein content with Bphi-1251 and R3177. These phages share a significant number of virion structural and genome replication genes and differ primarily in the complement of small accessory and unique proteins. On this basis, we have tentatively grouped these predominantly temperate Acinetobacter phages into a single cluster. However, we note that this assignment is not supported by the phylogenetic analysis of the portal and major capsid proteins. Further work is required to induce or isolate new relatives of these species before their taxonomy can be resolved in more detail.

Interestingly, all of the temperate Acinetobacter phages, whether induced from the host lysogens or isolated from environmental sources, exhibited a limited degree of similarity, ranging between $15-51 \%$ at the nucleotide sequence level and having $21-51 \%$ of shared proteins (Figure 8). These include morphologically distinct phages (Figure 9) comprising of sub-clusters I1 (AbTJ and Ab105-1phi), L1 (RPH2R), L2 (TRS2), and the singleton TRS1. 
L2

L1

F2

F5

Singleton

K1

K1

K1

(n)

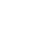

1

$$
\begin{array}{r}
\text { KT588074 Ab105-1phi } \\
1-41,496 \mathrm{bp}
\end{array}
$$

$$
\begin{array}{r}
\text { MK340941 AbTJ } \\
1-42,670 \mathrm{bp}
\end{array}
$$

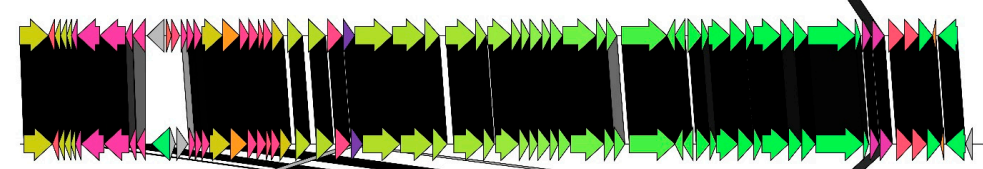

$$
\begin{array}{r}
\text { MH115576 AM106 } \\
1-52,031 \text { bp }
\end{array}
$$

MT361972 Ab11510-phi 1-50,916 bp

JX403940 Bphi-B1251
$1-45,364$ bp

KP861230 YMC-11-R3177
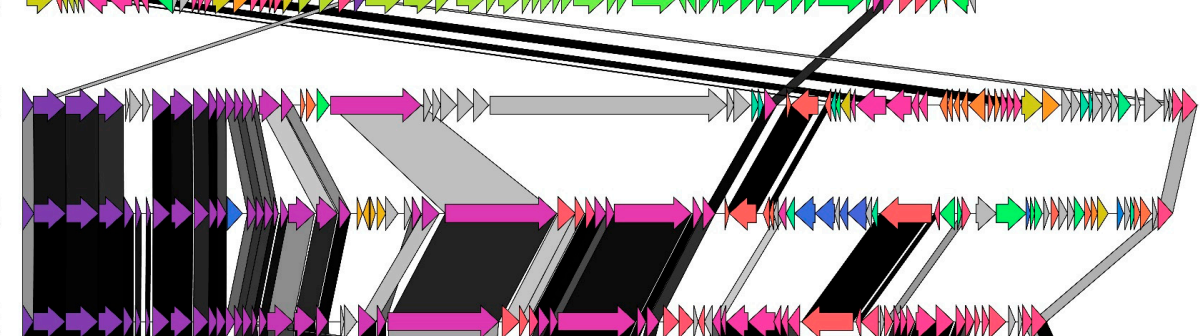

$1-47,575$

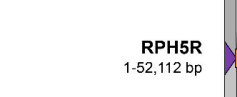

KT588073 Ab105-3phi $1-63,785 \mathrm{bp}$

KT588075 Ab105-2phi

$$
\begin{aligned}
& \text { Ab105-2phi } \\
& 1-61,304 \mathrm{bp}
\end{aligned}
$$

KX268652 vB_AbaS_TRS1

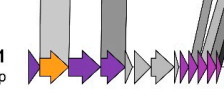

$$
\begin{array}{r}
\text { MT344105 fLi-Aba03 } \\
\text { 1-34,931 bp } \\
\text { MT344104 fLi-Aba02 } \\
\text { 1-35,093 bp } \\
\text { MT344103 fEg-Aba01 } \\
\text { 1-33,779 bp }
\end{array}
$$
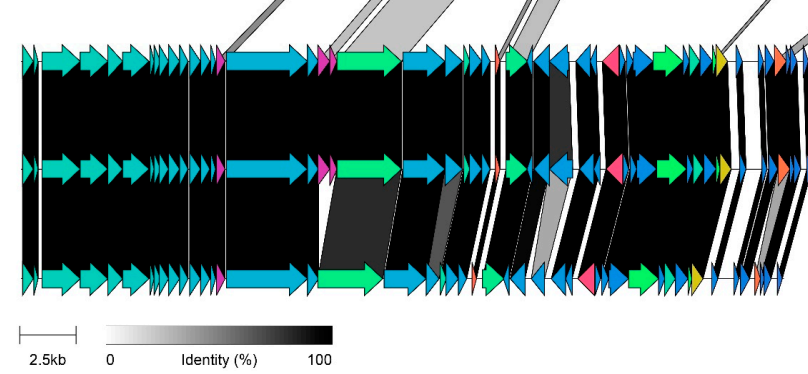

Figure 8. Comparative genome alignment of sub-clusters comprising of temperate phages infecting Acinetobacter sp. Phage genomes are presented alongside their designated sub-cluster, accession number, name and genome length. Coding sequences are represented by arrows, coloured to reflect homologous groups identified by Clinker, and are linked by grey bars shaded to represent the percentage amino acid identity, as indicated in the legend. 

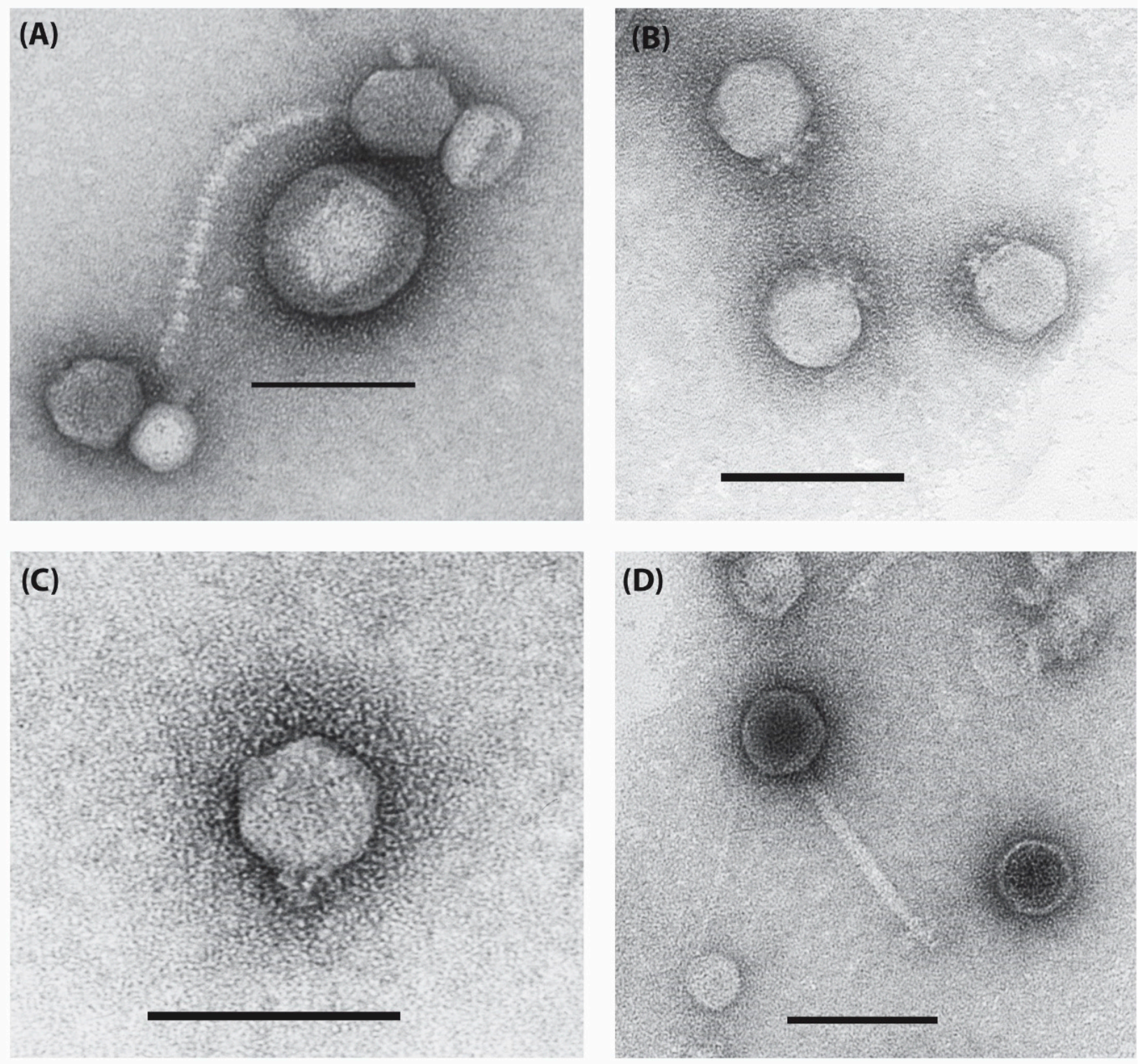

Figure 9. Novel temperate phages of A. baumannii. Transmission electron micrographs of (A) RPH5R; (B) RPH2R (C) TRS2; and (D) TRS1, negatively stained with $2 \% w / v$ uranyl acetate. Scale bars represent $100 \mathrm{~nm}$.

Preliminary searches conducted with BLASTn, using the temperate Acinetobacter phage genomes, demonstrates the presence of similar prophage regions in a number of sequenced $A$. baumannii isolates, though at varying levels of coverage and sequence identity. Most isolates of A. baumannii appear to be poly-lysogens, with one study reporting a median of seven prophages across 177 analysed genomes [35]. In a larger study of 795 A. baumannii genomes, Costa et al. identified 19 clusters of prophages with a genome identity above 50\% [36]. However, the impact of prophage carriage upon virulence, fitness, and metabolism in Acinetobacter lysogens has yet to be investigated in detail.

\subsection{New Strains in the Genus Lokivirus}

The genus Lokivirus is expanded to include an additional two strains of IME_AB3 D0 and Ab_SZ3. Cluster G currently contains five siphoviruses that possess modular, syntenic genomes of between 41.3 and $43 \mathrm{~kb}$ and share 43 core protein groups, which represent the gene products involved in genome replication, transcriptional regulation, and virion structural proteins (Figure 10). 


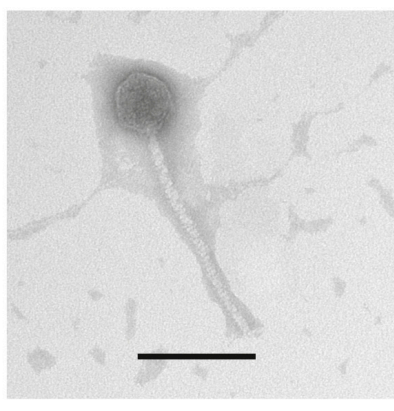

(B)

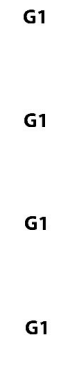

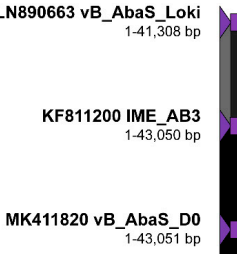

MW151244 Ab_SZ3

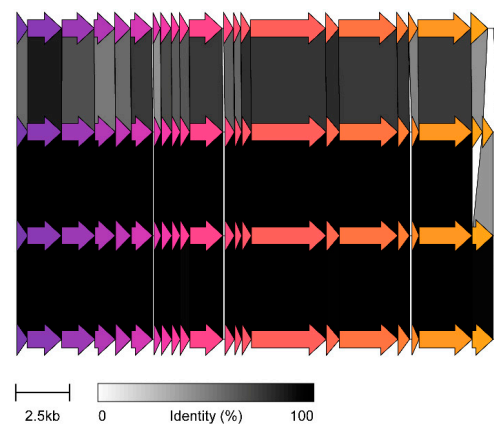

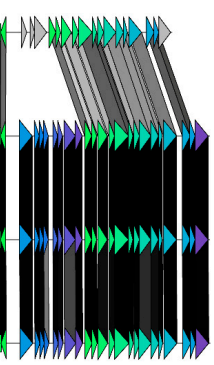

Figure 10. (A) Transmission electron micrograph of Acinetobacter phage Loki. The scale bar represents $100 \mathrm{~nm}$. (B) Comparative genome alignment of phages comprising sub-cluster G1. Phage genomes are presented alongside their designated sub-cluster, accession number, name and genome length. Coding sequences are represented by arrows, coloured to reflect homologous groups identified by Clinker, and are linked by grey bars shaded to represent the percentage amino acid identity, as indicated in the legend.

The two species are distinguished solely by differences in the structure of the endolysin and by the complement of genes in the predicted early expression module. Lipooligosaccharide has been implicated as the target cell surface receptor for the infection of $A$. baumannii ATCC 17978 by Loki as a stop mutation in lpxA, which is involved in the biosynthesis of lipid A, abolishes adsorption [37].

\subsection{Phages DMU1 and SH-Ab15497 Comprise a New Genus of Siphoviruses}

We propose the creation of a new cluster to group phages DMU1 and SH-Ab15479, which is supported by a nucleotide similarity of $92.6 \%$ and a total of $98.1 \%$ proteins shared across the two genomes. Both phages exhibit a Bradley B1 siphovirus morphology and a $43.4 \mathrm{~kb}$ genome with 53 gene products. Of the 53 core proteins, the majority (41 proteins) are only found within this sub-cluster. The 11 proteins shared with other Acinetobacter phages include seven virion structural proteins and four hypothetical proteins (Figure 11).
J1

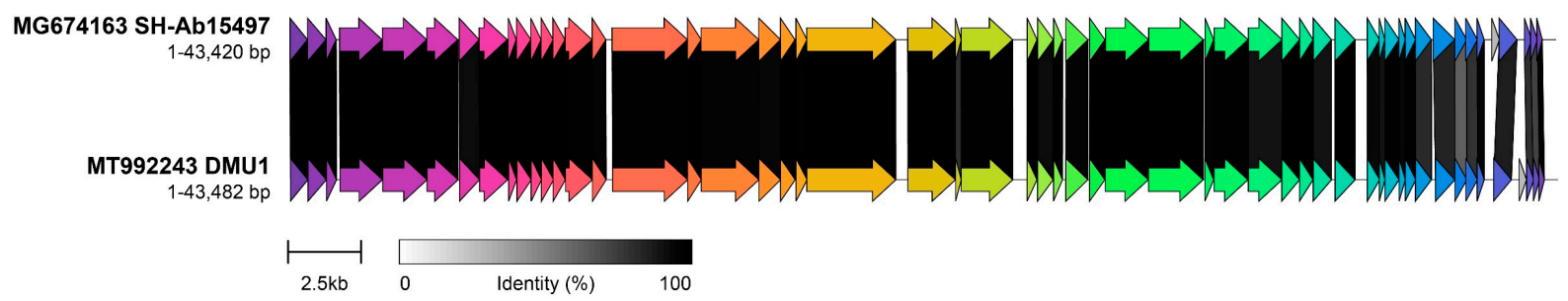

Figure 11. Comparative genome alignment of phages comprising sub-cluster J1. Phage genomes are presented alongside their designated sub-cluster, accession number, name and genome length. Coding sequences are represented by arrows, coloured to reflect homologous groups identified by Clinker, and are linked by grey bars shaded to represent the percentage amino acid identity, as indicated in the legend.

\subsection{Singleton Phages}

\subsubsection{Singletons Presley and XC38 Belong to the Family Schitoviridae}

Presley and XC38 are the only two representatives of the family Schitoviridae isolated to date that infect the species $A$. baumannii and A. pittii. The family is characterised by a podovirus morphology with members encapsulating linear genomes of $59-90 \mathrm{~kb}$ flanked by direct terminal repeats. A total of 17 proteins are conserved across all members of the family, including the characteristic virion-associated and two-subunit RNA polymerases [38]. Based on their limited degree of nucleotide similarity (11\%), Presley and XC38 are classified into two separate genera, Presleyvirus and Xceevirus. A total of 28 proteins $(33.7 \%)$ are 
shared between these two species, and, with the isolation of additional representative species, may support the creation of a new subfamily within the Schitoviridae (Figure 12).

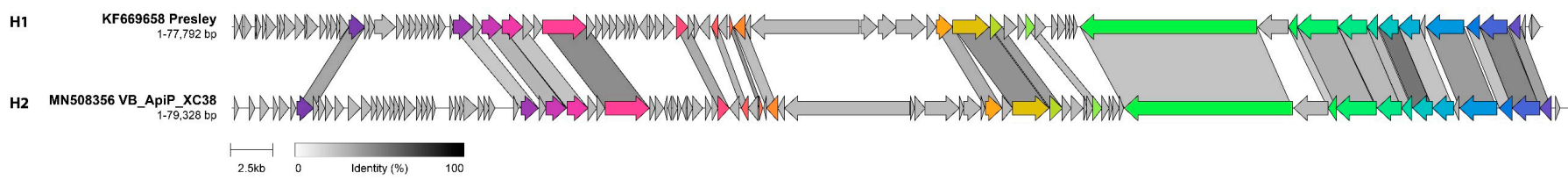

Figure 12. Comparative genome alignment of Acinetobacter phages classified within the family Schitoviridae. Phage genomes are presented alongside their designated sub-cluster, accession number, name and genome length. Coding sequences are represented by arrows, coloured to reflect homologous groups identified by Clinker, and are linked by grey bars shaded to represent the percentage amino acid identity, as indicated in the legend.

\subsubsection{Singletons BS46 and B9 Represent a New Subfamily of Myoviruses}

The myovirus BS46 was originally isolated in 1991 from sewage and used in an early assessment of the phage treatment of A. baumannii in a murine model [39]. BS46 was subsequently sequenced 25 years later, revealing a $94 \mathrm{~kb}$ linear genome with 176 predicted ORFs and three tRNAs [40]. B9 possesses a similarly sized genome of $93.6 \mathrm{~kb}$ and 168 predicted ORFs. Both B9 and BS46 form plaques surrounded by an opaque halo of reduced turbidity and encode structural proteins with capsule-specific depolymerase activity $[40,41]$. BS46 and B9 exhibited a 35\% nucleotide sequence similarity and share 79 (51\%) ORFs across the genome replication, virion structure, and assembly modules (Figure 13). Given the degree of similarity exhibited between these two phages, we propose that they are classified as separate genera within a new subfamily.

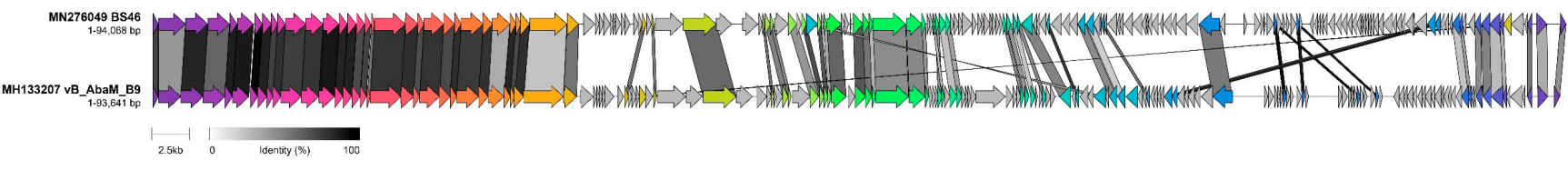

Figure 13. Comparative genome alignment of phages BS46 and B9. Phage genomes are presented alongside their designated sub-cluster, accession number, name and genome length. Coding sequences are represented by arrows, coloured to reflect homologous groups identified by Clinker, and are linked by grey bars shaded to represent the percentage amino acid identity, as indicated in the legend.

\subsubsection{Singleton ABPH49 Is a New Species of Vequintavirinae}

$\mathrm{ABPH} 49$ is the only representative of the subfamily Vequintavirinae known to infect A. baumannii and shows little sequence similarity to the existing phages in the extant sequence database. The relationship of ABPH49 to the Vequintavirinae is revealed by TBLASTX, where the genome exhibits approximately $40 \%$ coverage and $\sim 82 \%$ identity to members of the Cetrevirus. The $149.9 \mathrm{~kb}$ ABPH49 genome encodes 246 unique proteins and a further 32 that are shared with other Acinetobacter phages.

\subsubsection{Singleton SH-Ab 15599 Is a New Species of Ackermannviridae}

The singleton phage $\mathrm{SH}-\mathrm{Ab} 15599$ has an A1 myovirus morphology and a genome of $143.2 \mathrm{~kb}$ [42], where 127 of the 174 predicted ORFs are unique among the Acinetobacter phages. The vContact 2 network analysis places this phage within a cluster formed by the family Ackermannviridae. While SH-Ab15599 shows no discernible nucleotide sequence similarity to these phages, searches using TBLASTX reveals some similarity to members of the genus Kuttervirus ( $30 \%$ coverage and $45 \%$ identity). Like other members of the Ackermanniviridae, $\mathrm{SH}-\mathrm{Ab} 15599$ exhibits the distinctive terminal tail morphology of branched tail fibers, in which each branch is formed by a single tailspike, suggesting that it may represent a new genus within this family. 


\subsubsection{Arae Is a Genomic Singleton}

Arae is a Bradley morphotype B2 siphovirus with a club-like distal tail structure that was isolated from raw sewage after enrichment with the A. baumannii clinical strain UKA9 (Figure 14). Arae encapsulates a $49.7 \mathrm{~kb}$ genome, which, at the time of writing, represents a genomic orphan with no phages showing similarity in the extant nucleotide sequence database. A total of 55 of the 66 predicted ORFs are unique and only 11 proteins are shared with other Acinetobacter phages.

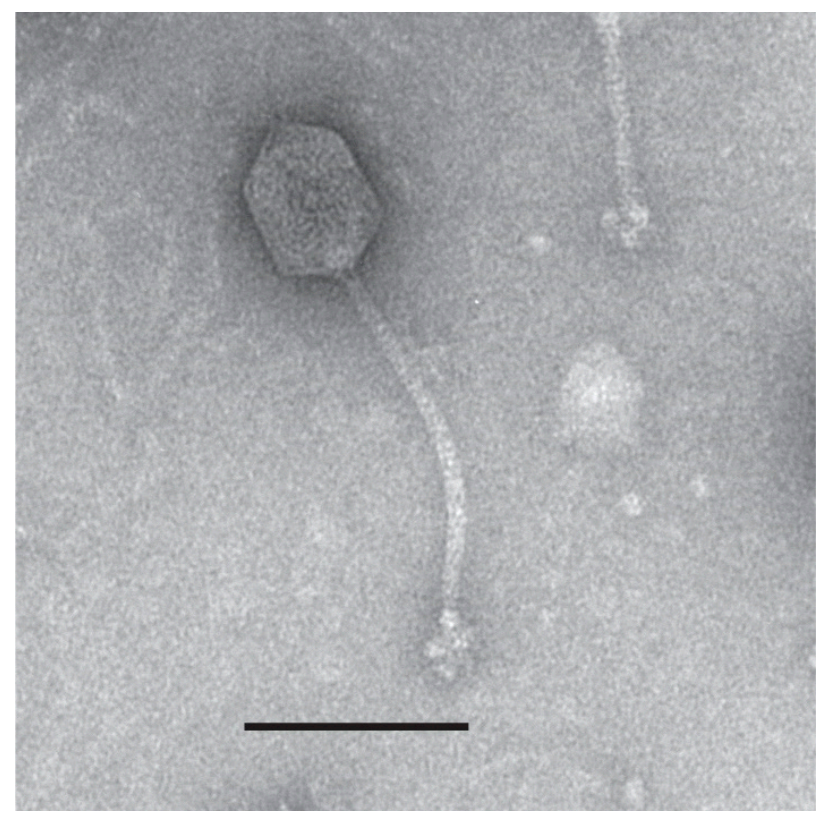

Figure 14. Transmission electron micrograph of phage Arae, negatively stained with $2 \% w / v$ uranyl acetate. The scale bar represents $100 \mathrm{~nm}$.

\subsubsection{ME3, TRS5 and BFG Are Unique 'Jumbo' Singleton Phages of Acinetobacter}

Recent metagenomics work has demonstrated that 'jumbo' phages, defined as those with genomes greater than $200 \mathrm{~kb}$ in size, are widely distributed in the environment [43]. To date, three jumbo phages have been isolated that infect Acinetobacter species, ME3, TRS5, and BFG.

ME3 was originally isolated from wastewater treatment effluent and encapsulates a $234.9 \mathrm{~kb}$ genome with 326 ORFs and 4 tRNAs. ME3 remains a genomic orphan, exhibiting no significant sequence similarity to the phages in the extant sequence database and is classified as the only member of the genus Metrivirus. The original isolate of ME3 has since been lost (C. Buttimer, personal communication). TRS5 and BFG are morphologically identical myoviruses (Figure 15) that were isolated from activated sludge and raw sewage from the same water treatment plant and propagate on A. baylyi ADP1 and A. baumannii UKA17, respectively. Despite the apparent morphological similarity, the genomes exhibit only a $17 \%$ nucleotide similarity. TRS5 and BFG encapsulate genomes of 371.5 and $378.1 \mathrm{~kb}$ with 661 and 666 predicted ORFs and $\geq 20$ tRNAs, respectively. These phages do not exhibit the modular organisation characteristic of smaller phage genomes. Instead, for TRS5 and $\mathrm{BFG}$, ORFs appear to be organised into operons, which are flanked by promoters and rhoindependent terminators. Despite the low level of sequence similarity, these two phages share a total of 214 proteins. A significant proportion $(>60 \%)$ of the genes encoded by these three phages are classified as unique, and the majority cannot be assigned predicted functions using current bioinformatics tools, suggesting that these phages represent distinct evolutionary lineages. 


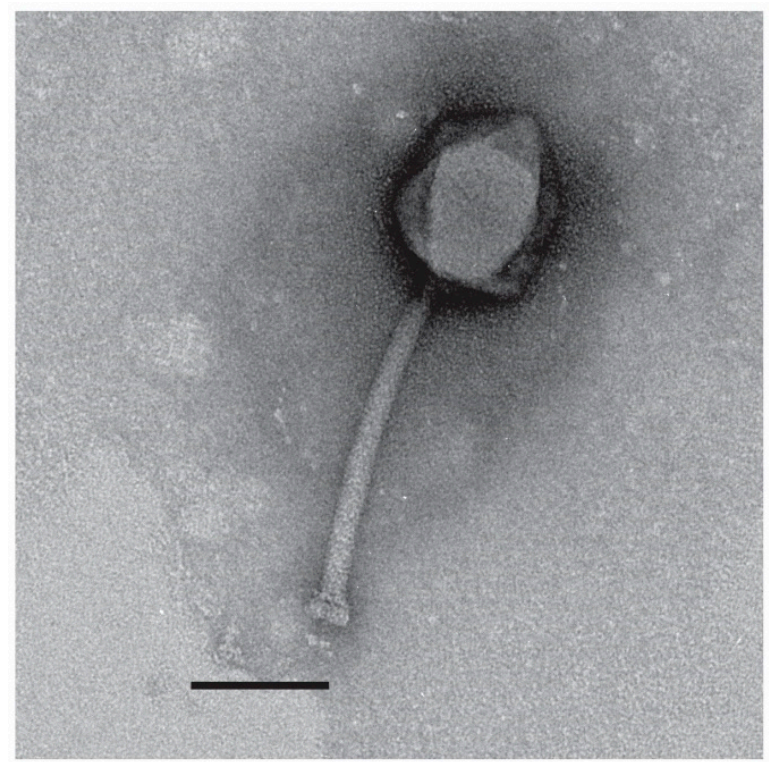

Figure 15. Transmission electron micrograph of the jumbo phage TRS5, stained with $2 \%$ w/v uranyl acetate. The scale bar represents $100 \mathrm{~nm}$.

\subsection{Select Features of Acinetobacter Phage Proteins}

The selection and development of phages for the knowledge-based application of phage therapy relies upon phenotypic as well as genomic data. Aside from the wellestablished criteria of avoiding phages that are temperate (unless genetically modified to prevent integration) and those that carry putative virulence factors or antibiotic resistance genes, the prediction of phenotype from genomic data-such as host specificity, resistance to host defense systems, and lysis mechanisms-may help to inform the selection of appropriate phages for clinical use or formulation within phage cocktails. To achieve this, it is vital to assess the biological role of Acinetobacter phage-derived proteins. Using the annotated pan-genome analysis, gene products involved in nucleotide modification, lysis, and virion structural depolymerases are discussed.

\subsubsection{Nucleotide Modification Systems}

Our analysis indicates that $39(29 \%)$ of the Acinetobacter phages encode methyltransferases. There are 13 phages that possess adenine-specific methyltransferases, while cytosine-specific methytransferases appear to be more widespread and are present in 28 genomes. While most encode a single methyltransferase, some phages possess two or more. For example, the phages BFG, TRS5, and 5W encode both adenine- and cytosinespecific methyltransferases. BS46 and BFG are each predicted to code for two distinct adenine-specific methyltransferases that exhibit little sequence similarity.

The InterProScan and $\mathrm{HH}$-suite results provided evidence for the presence of further gene products potentially involved in nucleotide modifications, including a 7-deazaguanine modification pathway in the jumbo phage TRS5. Recent work has shown the presence of a number of different 7-deazaguanine derivatives in phage genomes, including 2'deoxy-7-cyano-7-deazaguanosine ( $\left.\mathrm{dPreQ}_{0}\right), 2^{\prime}$-deoxy-7-amido-7-deazaguanosine (dADG), $2^{\prime}$-deoxy-7-aminomethyl-7-deazaguanosine (dPreQ1), and 2'-deoxyarchaeaosine $\left(\mathrm{dG}^{+}\right)$[44]. TRS5 encodes homologues to queC, queD, queE, queF, and $d p d A$ in a localised cluster of genes. A distally located folE-like GTP cyclohydrolase is also present in the genome.

SH-Ab15599 codes for two proteins containing an Alpha-glutamyl/putrescinyl thymine pyrophosphorylase clade 3 domain (InterPro: IPR041271) that is predicted to be involved in the catalysis of the hyper-modified bases alpha-glutamylthymine, putrescinylthymine, 5-(2-aminoethoxy)methyluridine, or 5-(2-aminoethyl)uridine. These types of modified pyrimidines have been shown to occur in several phage genomes, including $\Phi \mathrm{W}-14$ [45], SP10 [46], ViI [47], and M6 [47]. 
The sub-cluster K1 phages SH-Ab 15497 and DMU1 each encode a gene product with a predicted adenylosuccinate synthetase activity (IPR042109; group 911) that may be involved in purine biosynthesis. However, whether these gene products are involved in the synthesis of modified bases, as has been observed for the Cyanophage S-2L that incorporates 2,6-aminoadenine in place of adenine [48], remains to be determined.

In phages $\mathrm{T} 2, \mathrm{~T} 4$, and $\mathrm{T} 6$, the genomic DNA contains 5-hydroxymethylcytosine $\left({ }^{\mathrm{hm} 5} \mathrm{C}\right)$, which is further modified by glycosylation to yield glucosyl-5-methylcytosine. This process is catalysed by dCMP hydroxylmethytransferase (gene 42 in T4), yielding a hydroxymethylated cytosine monophosphate, which is then converted by T4 dNMP kinase to the diphosphate form. Following replication, ${ }^{\mathrm{hm} 5} \mathrm{C}$ is glucosylated by one of two DNA glucosyltransferases that produces an alpha or beta linkage between glucose and ${ }^{\mathrm{hm} 5} \mathrm{C}$ [48]. While homologues to a dCMP hydroxymethylase and T4 dNMP kinase were identified within the Twarogvirinae (Cluster A), we were unable to predict the presence of either alphaor beta glucosyltransferases using bioinformatics approaches, so whether these phages possess glucosylated DNA remains unknown.

\subsubsection{Lysis Cassettes}

The Acinetobacter phages have highly conserved endolysin and holin genes, with the most conserved present in 64 and 50 phages, respectively. About $81 \%$ are predicted to encode canonical holin-globular endolysins, $17 \%$ holin-modular endolysins, and $2 \%$ pinholin-SAR endolysins. The rare modular endolysin architecture is present only in the members of clusters B, I, F, and G, as well as in the singletons (e.g., 5W). In all cases, these proteins display an N-terminal Glyco_hydro_108 (PF05838.14) catalytic and a Cterminal PG_binding_3 (PF09374.12) domain. The Signal-arrest-release (SAR) endolysins are exclusively present in cluster $\mathrm{K}$, having an N-terminal transmembrane domain and a lysozyme catalytic domain (PF00959). Surprisingly, we did not identify proteins with spanin functions needed to disrupt the host outer membranes, suggesting that lysis cassettes lacking spanins may be a feature of Acinetobacter phages, which may have adopted an alternative mechanism to disrupt the outer membranes.

\subsubsection{Virion Structural Polysaccharide Depolymerases}

Virion structural depolymerases appear to be highly prevalent among the Acinetobacter viruses. From the 134 Acinetobacter phage genomes analysed, depolymerases were identified in 83 phages $(62 \%)$, indicating that the remaining phages $(38 \%)$ recognize hosts without enzymatic activity, either via LPS or outer membrane proteins. The most conserved depolymerase gene is shared by only 18 phages, demonstrating the vast diversity of these proteins. Although prevalent, depolymerase genes are exclusively located in clusters containing viruses with genomes of less than $90 \mathrm{~kb}$ (B, D, E, I, J, K, L, and M), and three singletons (SH-Ab15599, TRS1, and 5W). All members of these clusters encode depolymerases with no exceptions. For instance, while Autographiviridae viruses (cluster E) have genomes around $40 \mathrm{~kb}$, clusters $\mathrm{B}, \mathrm{D}, \mathrm{I}, \mathrm{J}, \mathrm{K}, \mathrm{L}$, and $\mathrm{M}$ contain viruses with myovirus and siphovirus morphotypes that also have genome sizes ranging between 40 to $90 \mathrm{~kb}$. By homology, 73 phages (54.5\%) contain capsular depolymerases, with the remaining $10(7.5 \%)$ encoding esterases-a class recently characterized in the Acinetobacter phage Aristophanes [49]. With the exception of the singleton SH-AB_15599, which codes for two adjacent capsular depolymerases, all viruses analysed here are predicted to code for a single depolymerase.

\section{Discussion}

As for many other host bacterial genera and species, there is significant diversity in the phages infecting Acinetobacter species. Since 2018, the number of sequenced Acinetobacter phage genomes available in the INSDC has expanded from 37 to 139. This number is expected to continue to increase given the importance of $A$. baumannii as a priority critical ESKAPE pathogen and the expanding interest in phage therapy. Our results demonstrate that these phages can be classified using nucleotide sequence identity, shared orthologous 
genes, and genomic organisation. The Acinetobacter phages are grouped into eight clusters (subfamilies), 38 subclusters (genera), and eight singleton genomes (single species genera). While the majority of core protein groups identified for each cluster represent genes that are coding for key products in genome replication, lysis, and virion structure and assembly, some core genes and numerous accessory protein groups of unknown function are located within the predicted early genome modules.

While the number of sequenced genomes of phages infecting Acinetobacter species has increased, little is still known about the underlying biology governing the subversion of host transcription, translation, and metabolism that facilitates a productive infection. Genes expressed early in phage infection have been associated with host takeover [50]. These early gene products have received increased attention for the identification and exploitation of novel antibacterial proteins and mechanisms of action [51]. Advances in RNA sequencing have allowed the temporal expression of phage genes across different timepoints in the infection cycle for a number of different phage-host combinations, including Yersinia [52], Vibrio [53], and Pseudomonas [54,55]. However, few early genes have been identified in Acinetobacter phage genomes. Instead, this status is often ascribed due to the position of genes within a genomic module. To date, only a single study has reported transcriptome data for infection in the A. baumannii strain AB1 with phages Abp1 (Cluster E) and AB1 (Cluster B) [56]. Given the diversity observed in the protein-coding genes of the Acinetobacter phages, it is likely that a variety of distinct mechanisms play a role in the early infection of host cells, some of which may be dependent upon the physiological status of the host cell (e.g., under conditions of nutrient limitation). A key challenge to furthering our understanding of productive infection in the Acinetobacter phages is to determine the functions of such early proteins, and to elucidate their roles and effects in the context of gene regulation, transcription, protein-protein interactions, and host cell metabolism. In addition to providing potential avenues for the identification of novel antibacterial mechanisms, such work might accommodate the need for tools in the genetic analysis and manipulation of this important pathogen.

Infections caused by carbapenem-resistant $A$. baumannii will continue to be of significant concern in the coming years. The WHO has highlighted that the current clinical pipeline still lacks novel antibiotics targeting this priority pathogen, and that those in development may be affected by cross-resistance given that most are derivatives of existing classes of antibiotics $[57,58]$. Given the increased interest in the application of phage therapy and use of phage-derived proteins, such as depolymerases and endolysins, it will be necessary to develop a platform that captures the important phenotypic features of individual phages, including host range and adsorption specificity, one step growth characteristics, and stability data, to supplement genomic information.

Many phages have adapted to encode tail fiber or tail spike proteins with enzymatic (depolymerase) activity to recognize and degrade the polysaccharides that decorate the bacterial cell surface $[59,60]$. The diversity of the capsular polysaccharides incurs a constant selective pressure on phages to evolve depolymerases with different specificities [61]. Current knowledge has shown that phage-derived depolymerases may degrade lipopolysaccharide (LPS) [62], capsule polymers [63], or modify surface polysaccharides through deacetylation [64]. However, only a small fraction of Acinetobacter phages have been reported to show depolymerase activity, and, to date, act predominantly upon capsular polysaccharides $[41,63,65,66]$.

Capsular depolymerases have been applied as a method to reduce $A$. baumannii virulence $[65,67]$. By stripping the cell surface capsular coats, depolymerases have been shown to render A. baumannii cells less virulent and more susceptible to the host immune system. As such, there is particular interest in understanding phage specificity for Acinetobacter capsular polysaccharides. To date, phages that are specific to 16 different capsular types of A. baumannii (K1-K2, K9, K19, K27, K32, K37-K38, K44-K45, K48, K87, K89, K91, K93, and $\mathrm{K} 116)$ have been described $[63,65,66,68-70]$. Here, we have shown that there is a large untapped source of depolymerases for Acinetobacter capsule types, which could be used 
as anti-virulence or anti-biofilm agents against $A$. baumannii infections. Elucidating the tertiary structures of the additional depolymerases could provide a structural framework by which to produce enzymes with broader specificity, or allow the engineering of phages with altered host specificity, as peformed in Klebsiella phages [71].

Phages of the class Caudoviricetes encode lysis cassettes to burst the host cell wall at the end of their lytic cycle in order to release progeny virions. Phages infecting Gram-negative bacteria are generally described with a holin-endolysin-spanin system. Holins act first to permeabilise the cytoplasmatic membrane to endolysins that then degrade the rigid peptidoglycan layer, resulting in osmotic lysis and cell death. Spanins are also used by phages, either as two-component or unimolecular proteins, to disrupt the outer membranes in this final step [72]. It was surprising to observe a lack of predicted spanins in the Acinetobacter phages. It is possible that these phages are using endolysins to interact with the outer membrane in the final step of the lytic cycle. A growing body of evidence has shown the ability of recombinant Acinetobacter phage endolysins to naturally degrade cells after exogenous application due to the presence of highly positively charged C-terminal domains with predicted amphipathic helical structures [73,74]. Another possibility might be as reported in the coliphage $\varphi \mathrm{KT}$ phage, which encodes an independent protein-encoding gene that disrupts the membrane using a mechanism similar to antimicrobial peptides [75].

Restriction-modification ( $\mathrm{R}-\mathrm{M})$ systems are perhaps the most well characterized resistance mechanisms against foreign DNA. These systems are widespread among prokaryotes and are present in $90 \%$ of bacterial genomes [76]. R-M systems are categorized into four main types: type I, II, III, and IV. Type II systems, consisting of a restriction endonuclease and cognate DNA adenine or cytosine methyltransferase, are the most abundant and the best studied so far [77]. In type II systems, the restriction endonuclease and methyltransferase both recognize the same specific target sequence. In the absence of methylation at the recognition site, the restriction enzyme cleaves the DNA. This acts as a defense mechanism against foreign DNA, such as phages. To counteract the bacterial R-M defense systems, phages have evolved several different strategies that include the synthesis of methyltransferases, production of anti-restriction proteins, and the use of a variety of modified bases [78]. Nucleotide modification appears to be relatively widespread among the Acinetobacter phages. A total of 39 phages were predicted to encode adenine or cytosine specific methyltransferases and three phages had gene products that may point to the presence of other types of modification, which includes a 7-deazaguanine derivative. Considering that methylation has been shown to have additional functions in bacteria, including roles in DNA replication, DNA repair, and regulation of transcription [79], it is possible that the presence of modified nucleosides in phage genomes may play a wider role in the infection cycle than just defence against restriction-modification systems. To date, no studies have specifically addressed the presence of modified bases in Acinetobacter phage genomes.

While the numbers and diversity of Acinetobacter phage genomes in the INSDC has increased, we still know little about the underlying biology around both the mechanisms of productive infection by lytic phages and the effects of prophage carriage upon the lysogen in this host genus. While this work provides an updated framework to aid in the annotation and classification of newly isolated Acinetobacter phages, it is clear that more applied research is required to supplement the information derived from the in silico analysis presented here.

Supplementary Materials: The following are available online at https:/ / www.mdpi.com/article/ 10.3390/v14020181/s1, Figure S1: VIRIDC analysis of 139 Acinetobacter phages, Table S1: Acinetobacter bacteriophage genomes deposited in GenBank on the 21 January 2021, File S1: Pan-genome analysis and annotation of proteins encoded by 139 Acinetobacter phages. 
Author Contributions: Conceptualization, D.T. and H.O.; methodology, D.T. and H.O.; formal analysis, D.T., R.D. and H.O.; writing—original draft preparation, D.T. and H.O.; writing-review and editing, D.T., H.O., J.M.S., E.M.A. and B.E.; visualization, D.T. and H.O. All authors have read and agreed to the published version of the manuscript.

Funding: H.O. was funded by the Portuguese Foundation for Science and Technology, grant number UIDB/04469/2020. E.M.A was funded by the UK Biotechnology and Biological Sciences Research Council (BBSRC) Institute Strategic Program grant BB/R012490/1 to the Gut Microbes and Health Research Programme and its constituent projects BBS/E/F/000PR10353 and BBS/E/F/000PR10356.

Institutional Review Board Statement: Not applicable.

Informed Consent Statement: Not applicable.

Data Availability Statement: Not applicable.

Conflicts of Interest: The authors declare no conflict of interest. The funders had no role in the design of the study; in the collection, analyses, or interpretation of data; in the writing of the manuscript, or in the decision to publish the results.

\section{References}

1. Yutin, N.; Makarova, K.S.; Gussow, A.B.; Krupovic, M.; Segall, A.; Edwards, R.A.; Koonin, E.V. Discovery of an expansive bacteriophage family that includes the most abundant viruses from the human gut. Nat. Microbiol. 2018, 3, 38-46. [CrossRef] [PubMed]

2. Adriaenssens, E.M. Phage diversity in the human gut microbiome: A taxonomist's perspective. MSystems 2021, 6, e0079921. [CrossRef] [PubMed]

3. Adriaenssens, E.M.; Brister, J.R. How to name and classify your phage: An informal guide. Viruses 2017, 9, 70. [CrossRef]

4. Kyriakidis, I.; Vasileiou, E.; Pana, Z.D.; Tragiannidis, A. Acinetobacter baumannii antibiotic resistance mechanisms. Pathogens 2021, 10, 373. [CrossRef] [PubMed]

5. Potron, A.; Poirel, L.; Nordmann, P. Emerging broad-spectrum resistance in Pseudomonas aeruginosa and Acinetobacter baumannii: Mechanisms and epidemiology. Int. J. Antimicrob. Agents 2015, 45, 568-585. [CrossRef]

6. Mea, H.J.; Yong, P.V.C.; Wong, E.H. An overview of Acinetobacter baumannii pathogenesis: Motility, adherence and biofilm formation. Microbiol. Res. 2021, 247, 126722. [CrossRef] [PubMed]

7. Almasaudi, S.B. Acinetobacter spp. as nosocomial pathogens: Epidemiology and resistance features. Saudi J. Biol. Sci. 2018, 25, 586-596. [CrossRef] [PubMed]

8. Nemec, A.; Krizova, L.; Maixnerova, M.; van der Reijden, T.J.K.; Deschaght, P.; Passet, V.; Vaneechoutte, M.; Brisse, S.; Dijkshoorn, L. Genotypic and phenotypic characterization of the Acinetobacter calcoaceticus-Acinetobacter baumannii complex with the proposal of Acinetobacter pittii sp. nov (formerly Acinetobacter genomic species 3) and Acinetobacter nosocomialis sp. nov (formerly Acinetobacter genomic species 13TU). Res. Microbiol. 2011, 162, 393-404. [CrossRef]

9. Rodrigues, D.L.N.; Morais-Rodrigues, F.; Hurtado, R.; dos Santos, R.G.; Costa, D.C.; Barh, D.; Ghosh, P.; Alzahrani, K.J.; Soares, S.C.; Ramos, R.; et al. Pan-resistome insights into the multidrug resistance of Acinetobacter baumannii. Antibiotics 2021, 10, 596. [CrossRef]

10. Alvarez, V.E.; Quiroga, M.P.; Galan, A.V.; Vilacoba, E.; Quiroga, C.; Ramirez, M.S.; Centron, D. Crucial role of the accessory genome in the evolutionary trajectory of Acinetobacter baumannii global clone 1. Front. Microbiol. 2020, 11, 342. [CrossRef]

11. Holt, K.; Kenyon, J.J.; Hamidian, M.; Schultz, M.B.; Pickard, D.J.; Dougan, G.; Hall, R. Five decades of genome evolution in the globally distributed, extensively antibiotic-resistant Acinetobacter baumannii global clone 1. Microb. Genom. 2016, 2, e000052. [CrossRef]

12. Vahhabi, A.; Hasani, A.; Rezaee, M.A.; Baradaran, B.; Samadi Kafil, H.; Abbaszadeh, F.; Dehghani, L. A plethora of carbapenem resistance in Acinetobacter baumannii: No end to a long insidious genetic journey. J. Chemother. 2021, 33, 137-155. [CrossRef]

13. Turner, D.; Ackermann, H.W.; Kropinski, A.M.; Lavigne, R.; Sutton, J.M.; Reynolds, D.M. Comparative analysis of 37 Acinetobacter bacteriophages. Viruses 2017, 10, 5. [CrossRef]

14. Seemann, T. Prokka: Rapid prokaryotic genome annotation. Bioinformatics 2014, 30, 2068-2069. [CrossRef] [PubMed]

15. Grazziotin, A.L.; Koonin, E.V.; Kristensen, D.M. Prokaryotic virus orthologous groups (pVOGs): A resource for comparative genomics and protein family annotation. Nucleic Acids Res. 2017, 45, D491-D498. [CrossRef] [PubMed]

16. Cook, R.; Brown, N.; Redgwell, T.; Rihtman, B.; Barnes, M.; Clokie, M.; Stekel, D.J.; Hobman, J.; Jones, M.A.; Millard, A. INfrastructure for a PHAge REference Database: Identification of large-scale biases in the current collection of phage genomes. Ther. Appl. Res. 2021, 2, 214-223. [CrossRef]

17. Shannon, P.; Markiel, A.; Ozier, O.; Baliga, N.S.; Wang, J.T.; Ramage, D.; Amin, N.; Schwikowski, B.; Ideker, T. Cytoscape: A software environment for integrated models of biomolecular interaction networks. Genome Res. 2003, 13, 2498-2504. [CrossRef] [PubMed] 
18. Moraru, C.; Varsani, A.; Kropinski, A.M. VIRIDC-A novel tool to calculate the intergenomic similarities of prokaryote-infecting viruses. Viruses 2020, 12, 1268. [CrossRef]

19. Bayliss, S.C.; Thorpe, H.A.; Coyle, N.M.; Sheppard, S.K.; Feil, E.J. PIRATE: A fast and scalable pangenomics toolbox for clustering diverged orthologues in bacteria. Gigascience 2019, 8, giz119. [CrossRef]

20. Jones, P.; Binns, D.; Chang, H.Y.; Fraser, M.; Li, W.; McAnulla, C.; McWilliam, H.; Maslen, J.; Mitchell, A.; Nuka, G.; et al. Interproscan 5: Genome-scale protein function classification. Bioinformatics 2014, 30, 1236-1240. [CrossRef] [PubMed]

21. Sievers, F.; Higgins, D.G. Clustal Omega for making accurate alignments of many protein sequences. Protein Sci. 2018, 27, 135-145. [CrossRef] [PubMed]

22. Cresawn, S.G.; Bogel, M.; Day, N.; Jacobs-Sera, D.; Hendrix, R.W.; Hatfull, G.F. Phamerator: A bioinformatic tool for comparative bacteriophage genomics. BMC Bioinform. 2011, 12, 395. [CrossRef]

23. Huson, D.H.; Bryant, D. Application of phylogenetic networks in evolutionary studies. Mol. Biol. Evol. 2006, $23,254-267$. [CrossRef] [PubMed]

24. Nguyen, L.T.; Schmidt, H.A.; von Haeseler, A.; Minh, B.Q. IQ-TREE: A fast and effective stochastic algorithm for estimating maximum-likelihood phylogenies. Mol. Biol. Evol. 2015, 32, 268-274. [CrossRef] [PubMed]

25. Minh, B.Q.; Schmidt, H.A.; Chernomor, O.; Schrempf, D.; Woodhams, M.D.; von Haeseler, A.; Lanfear, R. IQ-TREE 2: New models and efficient methods for phylogenetic inference in the genomic era. Mol. Biol. Evol. 2020, 37, 1530-1534. [CrossRef]

26. Hoang, D.T.; Chernomor, O.; von Haeseler, A.; Minh, B.Q.; Vinh, L.S. UFBoot2: Improving the ultrafast bootstrap approximation. Mol. Biol. Evol. 2018, 35, 518-522. [CrossRef] [PubMed]

27. Kalyaanamoorthy, S.; Minh, B.Q.; Wong, T.K.F.; von Haeseler, A.; Jermiin, L.S. ModelFinder: Fast model selection for accurate phylogenetic estimates. Nat. Methods 2017, 14, 587-589. [CrossRef] [PubMed]

28. Letunic, I.; Bork, P. Interactive Tree Of Life (iTOL) v5: An online tool for phylogenetic tree display and annotation. Nucleic Acids Res. 2021, 49, W293-W296. [CrossRef]

29. Gilchrist, C.L.M.; Chooi, Y.H. Clinker \& clustermap. js: Automatic generation of gene cluster comparison figures. Bioinformatics 2021, 37, 2473-2475. [CrossRef]

30. Turner, D.; Kropinski, A.M.; Adriaenssens, E.M. A roadmap for genome-based phage taxonomy. Viruses 2021, 13, 506. [CrossRef] [PubMed]

31. Merabishvili, M.; Vandenheuvel, D.; Kropinski, A.M.; Mast, J.; De Vos, D.; Verbeken, G.; Noben, J.P.; Lavigne, R.; Vaneechoutte, M.; Pirnay, J.P. Characterization of newly isolated lytic bacteriophages active against Acinetobacter baumannii. PLoS ONE 2014, mboxemph9, e104853. [CrossRef]

32. Essoh, C.; Vernadet, J.P.; Vergnaud, G.; Coulibaly, A.; Kakou-N'Douba, A.; N'Guetta, A.S.; Resch, G.; Pourcel, C. Complete genome sequences of five Acinetobacter baumannii phages from Abidjan, Côte d'ivoire. Microbiol. Resour. Announc. 2019, 8, e01358-18. [CrossRef]

33. Jeon, J.; Park, J.H.; Yong, D. Efficacy of bacteriophage treatment against carbapenem-resistant Acinetobacter baumannii in Galleria mellonella larvae and a mouse model of acute pneumonia. BMC Microbiol. 2019, 19, 70. [CrossRef]

34. Popova, A.V.; Shneider, M.M.; Myakinina, V.P.; Bannov, V.A.; Edelstein, M.V.; Rubalskii, E.O.; Aleshkin, A.V.; Fursova, N.K.; Volozhantsev, N.V. Characterization of myophage AM24 infecting Acinetobacter baumannii of the K9 capsular type. Arch. Virol. 2019, 164, 1493-1497. [CrossRef]

35. Loh, B.; Chen, J.; Manohar, P.; Yu, Y.; Hua, X.; Leptihn, S. A biological inventory of prophages in A. baumannii genomes reveal distinct distributions in classes, length, and genomic positions. Front. Microbiol. 2020, 11, 579802. [CrossRef]

36. Costa, A.R.; Monteiro, R.; Azeredo, J. Genomic analysis of Acinetobacter baumannii prophages reveals remarkable diversity and suggests profound impact on bacterial virulence and fitness. Sci. Rep. 2018, 8, 15346. [CrossRef]

37. Turner, D.; Wand, M.E.; Briers, Y.; Lavigne, R.; Sutton, J.M.; Reynolds, D.M. Characterisation and genome sequence of the lytic Acinetobacter baumannii bacteriophage vB_AbaS_Loki. PLoS ONE 2017, 12, e0172303. [CrossRef]

38. Wittmann, J.; Turner, D.; Millard, A.D.; Mahadevan, P.; Kropinski, A.M.; Adriaenssens, E.M. From orphan phage to a proposed new family-the diversity of N4-like viruses. Antibiotics 2020, 9, 663. [CrossRef]

39. Soothill, J.S. Treatment of experimental infections of mice with bacteriophages. J. Med. Microbiol. 1992, 37, 258-261. [CrossRef]

40. Popova, A.V.; Shneider, M.M.; Mikhailova, Y.V.; Shelenkov, A.A.; Shagin, D.A.; Edelstein, M.V.; Kozlov, R.S. Complete genome sequence of Acinetobacter baumannii phage BS46. Microbiol. Resour. Announc. 2020, 9, e00398-20. [CrossRef]

41. Oliveira, H.; Costa, A.R.; Ferreira, A.; Konstantinides, N.; Santos, S.B.; Boon, M.; Noben, J.P.; Lavigne, R.; Azeredo, J. Functional analysis and antivirulence properties of a new depolymerase from a myovirus that infects Acinetobacter baumannii capsule K45. J. Virol. 2019, 93, e01163-18. [CrossRef] [PubMed]

42. Hua, Y.F.; Luo, T.T.; Yang, Y.Q.; Dong, D.; Wang, R.; Wang, Y.J.; Xu, M.S.; Guo, X.K.; Hu, F.P.; He, P. Phage therapy as a promising new treatment for lung infection caused by carbapenem-resistant Acinetobacter baumannii in mice. Front. Microbiol. 2018, 8, 2659. [CrossRef] [PubMed]

43. Al-Shayeb, B.; Sachdeva, R.; Chen, L.X.; Ward, F.; Munk, P.; Devoto, A.; Castelle, C.J.; Olm, M.R.; Bouma-Gregson, K.; Amano, Y.; et al. Clades of huge phages from across earth's ecosystems. Nature 2020, 578, 425-431. [CrossRef]

44. Hutinet, G.; Kot, W.; Cui, L.; Hillebrand, R.; Balamkundu, S.; Gnanakalai, S.; Neelakandan, R.; Carstens, A.B.; Fa Lui, C.; Tremblay, D.; et al. 7-deazaguanine modifications protect phage DNA from host restriction systems. Nat. Commun. 2019, 10, 5442. [CrossRef] 
45. Kropinski, A.M.; Bose, R.J.; Warren, R.A. 5-(4-aminobutylaminomethyl)uracil, an unusual pyrimidine from the deoxyribonucleic acid of bacteriophage phiW-14. Biochemistry 1973, 12, 151-157. [CrossRef]

46. Witmer, H. Synthesis of deoxythymidylate and the unusual deoxynucleotide in mature DNA of Bacillus subtilis bacteriophage SP10 occurs by postreplicational modification of 5-hydroxymethyldeoxyuridylate. J. Virol. 1981, 39, 536-547. [CrossRef]

47. Lee, Y.J.; Dai, N.; Walsh, S.E.; Muller, S.; Fraser, M.E.; Kauffman, K.M.; Guan, C.; Correa, I.R., Jr.; Weigele, P.R. Identification and biosynthesis of thymidine hypermodifications in the genomic DNA of widespread bacterial viruses. Proc. Natl. Acad. Sci. USA 2018, 115, E3116-E3125. [CrossRef] [PubMed]

48. Weigele, P.; Raleigh, E.A. Biosynthesis and function of modified bases in bacteria and their viruses. Chem. Rev. 2016, 116, 12655-12687. [CrossRef] [PubMed]

49. Timoshina, O.Y.; Shneider, M.M.; Evseev, P.V.; Shchurova, A.S.; Shelenkov, A.A.; Mikhaylova, Y.V.; Sokolova, O.S.; Kasimova, A.A.; Arbatsky, N.P.; Dmitrenok, A.S.; et al. Novel Acinetobacter baumannii bacteriophage Aristophanes encoding structural polysaccharide deacetylase. Viruses 2021, 13, 1688. [CrossRef] [PubMed]

50. Roucourt, B.; Lavigne, R. The role of interactions between phage and bacterial proteins within the infected cell: A diverse and puzzling interactome. Environ. Microbiol. 2009, 11, 2789-2805. [CrossRef] [PubMed]

51. Wan, X.; Hendrix, H.; Skurnik, M.; Lavigne, R. Phage-based target discovery and its exploitation towards novel antibacterial molecules. Curr. Opin. Biotechnol. 2021, 68, 1-7. [CrossRef] [PubMed]

52. Leskinen, K.; Blasdel, B.G.; Lavigne, R.; Skurnik, M. RNA-sequencing reveals the progression of phage-host interactions between $\phi$ R1-37 and Yersinia enterocolitica. Viruses 2016, 8, 111. [CrossRef] [PubMed]

53. Luo, P.; Lui, Q.; Deng, Y.; Tian, Y.; Yun, L.; Hu, C. Strand-specific RNA-Seq analysis provides first insight into transcriptome response of Vibrio alginolyticus to phage infection. Mar. Genom. 2018, 38, 5-8. [CrossRef]

54. Blasdel, B.G.; Chevallereau, A.; Monot, M.; Lavigne, R.; Debarbieux, L. Comparative transcriptomics analyses reveal the conservation of an ancestral infectious strategy in two bacteriophage genera. ISME J. 2017, 11, 1988-1996. [CrossRef]

55. Wicke, L.; Ponath, P.; Coppens, L.; Gerovac, M.; Lavigne, R.; Vogel, J. Introducing differential RNA-seq mapping to track the early infection phase for Pseudomonas phage $\phi \mathrm{KZ}$. RNA Biol. 2021, 18, 1099-1110. [CrossRef]

56. Yang, Z.C.; Yi, S.P.; Li, G.; Wang, J.; Huang, G.T.; Jiang, B.; You, B.; Gong, Y.L.; Zhang, C.; Luo, X.Q.; et al. Global transcriptomic analysis of the interactions between phage phi abp1 and extensively drug-resistant Acinetobacter baumannii. Msystems 2019, 4, e00068-19. [CrossRef]

57. Theuretzbacher, U.; Bush, K.; Harbarth, S.; Paul, M.; Rex, J.H.; Tacconelli, E.; Thwaites, G.E. Critical analysis of antibacterial agents in clinical development. Nat. Rev. Microbiol. 2020, 18, 286-298. [CrossRef]

58. WHO. 2020 Antibacterial Agents in Clinical and Preclinical Development: An Overview and Analysis; World Health Organization: Geneva, Switzerland, 2021.

59. Knecht, L.E.; Veljkovic, M.; Fieseler, L. Diversity and function of phage encoded depolymerases. Front. Microbiol. 2019, 10, 2949. [CrossRef] [PubMed]

60. Singh, J.K.; Adams, F.G.; Brown, M.H. Diversity and function of capsular polysaccharide in Acinetobacter baumannii. Front. Microbiol. 2018, 9, 3301. [CrossRef]

61. Santos, S.B.; Melo, L.D.R.; Oliveira, H. Phage structural antimicrobial proteins. In Exploitation for Biocontrol and Therapeutics; Coffey, A., Buttimer, C., Eds.; Caister Academic: Poole, UK, 2020; pp. 419-476.

62. Greenfield, J.; Shang, X.; Luo, H.; Zhou, Y.; Heselpoth, R.D.; Nelson, D.C.; Herzberg, O. Structure and tailspike glycosidase machinery of ORF212 from E. coli O157:H7 phage CBA120 (TSP3). Sci. Rep. 2019, 9, 7349. [CrossRef]

63. Oliveira, H.; Costa, A.R.; Konstantinides, N.; Ferreira, A.; Akturk, E.; Sillankorva, S.; Nemec, A.; Shneider, M.; Dotsch, A.; Azeredo, J. Ability of phages to infect Acinetobacter calcoaceticus-Acinetobacter baumannii complex species through acquisition of different pectate lyase depolymerase domains. Environ. Microbiol. 2017, 19, 5060-5077. [CrossRef]

64. Prokhorov, N.S.; Riccio, C.; Zdorovenko, E.L.; Shneider, M.M.; Browning, C.; Knirel, Y.A.; Leiman, P.G.; Letarov, A.V. Function of bacteriophage G7C esterase tailspike in host cell adsorption. Mol. Microbiol. 2017, 105, 385-398. [CrossRef] [PubMed]

65. Oliveira, H.; Mendes, A.; Fraga, A.G.; Ferreira, A.; Pimenta, A.I.; Mil-Homens, D.; Fialho, A.M.; Pedrosa, J.; Azeredo, J. K2 capsule depolymerase is highly stable, is refractory to resistance, and protects larvae and mice from Acinetobacter baumannii sepsis. Appl. Environ. Microbiol. 2019, 85, e00934-19. [CrossRef]

66. Popova, A.V.; Lavysh, D.G.; Klimuk, E.I.; Edelstein, M.V.; Bogun, A.G.; Shneider, M.M.; Goncharov, A.E.; Leonov, S.V.; Severinov, K.V. Novel Fri1-like viruses infecting Acinetobacter baumannii-vB_AbaP_AS11 and vB_AbaP_AS12-characterization, comparative genomic analysis, and host-recognition strategy. Viruses 2017, 9, 188. [CrossRef]

67. Liu, Y.; Leung, S.S.Y.; Guo, Y.; Zhao, L.; Jiang, N.; Mi, L.; Li, P.; Wang, C.; Qin, Y.; Mi, Z.; et al. The capsule depolymerase Dpo48 rescues Galleria mellonella and mice from Acinetobacter baumannii systemic infections. Front. Microbiol. 2019, 10, 545. [CrossRef]

68. Popova, A.V.; Shneider, M.M.; Arbatsky, N.P.; Kasimova, A.A.; Senchenkova, S.N.; Shashkov, A.S.; Dmitrenok, A.S.; Chizhov, A.O.; Mikhailova, Y.V.; Shagin, D.A.; et al. Specific interaction of novel Friunavirus phages encoding tailspike depolymerases with corresponding Acinetobacter baumannii capsular types. J. Virol. 2020, 95, e01714-20. [CrossRef]

69. Knirel, Y.A.; Shneider, M.M.; Popova, A.V.; Kasimova, A.A.; Senchenkova, S.N.; Shashkov, A.S.; Chizhov, A.O. Mechanisms of Acinetobacter baumannii capsular polysaccharide cleavage by phage depolymerases. Biochemistry 2020, 85, 567-574. [CrossRef] 
70. Domingues, R.; Barbosa, A.; Santos, S.B.; Pires, D.P.; Save, J.; Resch, G.; Azeredo, J.; Oliveira, H. Unpuzzling Friunavirus-host interactions one piece at a time: Phage recognizes Acinetobacter pittii via a new k38 capsule depolymerase. Antibiotics 2021, 10, 1304. [CrossRef] [PubMed]

71. Latka, A.; Lemire, S.; Grimon, D.; Dams, D.; Maciejewska, B.; Lu, T.; Drulis-Kawa, Z.; Briers, Y. Engineering the modular receptor-binding proteins of Klebsiella phages switches their capsule serotype specificity. Mbio 2021, 12, e00455-21. [CrossRef] [PubMed]

72. Kongari, R.; Rajaure, M.; Cahill, J.; Rasche, E.; Mijalis, E.; Berry, J.; Young, R. Phage spanins: Diversity, topological dynamics and gene convergence. BMC Bioinform. 2018, 19, 326. [CrossRef] [PubMed]

73. Lai, M.J.; Lin, N.T.; Hu, A.; Soo, P.C.; Chen, L.K.; Chen, L.H.; Chang, K.C. Antibacterial activity of Acinetobacter baumannii phage $\phi \mathrm{AB} 2$ endolysin (LysAB2) against both gram-positive and gram-negative bacteria. Appl. Microbiol. Biotechnol. 2011, 90, 529-539. [CrossRef] [PubMed]

74. Lood, R.; Winer, B.Y.; Pelzek, A.J.; Diez-Martinez, R.; Thandar, M.; Euler, C.W.; Schuch, R.; Fischetti, V.A. Novel phage lysin capable of killing the multidrug-resistant gram-negative bacterium Acinetobacter baumannii in a mouse bacteremia model. Antimicrob. Agents Chemother. 2015, 59, 1983-1991. [CrossRef] [PubMed]

75. Holt, A.; Cahill, J.; Ramsey, J.; Martin, C.; O’Leary, C.; Moreland, R.; Maddox, L.T.; Galbadage, T.; Sharan, R.; Sule, P.; et al. Phage-encoded cationic antimicrobial peptide required for lysis. J. Bacteriol. 2021, JB0021421. [CrossRef] [PubMed]

76. Oliveira, P.H.; Touchon, M.; Rocha, E.P. The interplay of restriction-modification systems with mobile genetic elements and their prokaryotic hosts. Nucleic Acids Res. 2014, 42, 10618-10631. [CrossRef] [PubMed]

77. Pingoud, A.; Wilson, G.G.; Wende, W. Type II restriction endonucleases-a historical perspective and more. Nucleic Acids Res. 2014, 42, 7489-7527. [CrossRef] [PubMed]

78. Loenen, W.A.; Dryden, D.T.; Raleigh, E.A.; Wilson, G.G.; Murray, N.E. Highlights of the DNA cutters: A short history of the restriction enzymes. Nucleic Acids Res. 2014, 42, 3-19. [CrossRef] [PubMed]

79. Sanchez-Romero, M.A.; Casadesus, J. The bacterial epigenome. Nat. Rev. Microbiol. 2020, 18, 7-20. [CrossRef] 